\title{
Die professionelle pädagogische Beziehung zwischen Referendar*innen und ihren Seminarlehrkräften: Belastungsfaktor oder Ressource?
}

\author{
Tobias Kärner (D) - Michael Goller (D) - Caroline Bonnes (D) • \\ Elisabeth Maué ii
}

Eingegangen: 9. Dezember 2020 / Überarbeitet: 21. Juni 2021 / Angenommen: 9. September 2021 /

Online publiziert: 22. Februar 2022

(C) Der/die Autor(en) 2022

Zusammenfassung Der Beitrag befasst sich mit der professionellen pädagogischen Beziehung zwischen Referendar*innen und ihren Seminarlehrkräften, deren Beitrag für die Beanspruchung der Referendar*innen bisher noch nicht eingängig untersucht wurde. Das Ziel der Studie ist es, anhand einer Querschnittserhebung von 2583 Referendar*innen und ausgebildeten Lehrkräften, kompensatorische sowie verstärkende Effekte der Beziehungsdimensionen Transparenz, Fairness, Vertrauen und Ambivalenz auf die wahrgenommene Beanspruchung im Referendariat zu untersuchen. Die Analyse erfolgt mittels eines latent moderierten Strukturgleichungsansatzes. Die Ergebnisse zeigen, dass Belastungen durch die Kerntätigkeiten im Referendariat sowie Belastungen durch den Umgang mit Kolleg*innen im Lehrer*innenkollegium

Prof. Dr. Tobias Kärner $(\bowtie)$

Professur für Wirtschaftspädagogik, insbesondere Lehr- und Lernprozesse (560A), Universität

Hohenheim, Fruwirthstr. 47, 70599 Stuttgart, Deutschland

E-Mail: tobias.kaerner@uni-hohenheim.de

Dr. Michael Goller

Arbeitsgruppe Erziehungswissenschaft mit dem Schwerpunkt Bildungsmanagement und Bildungsforschung in der Weiterbildung, Universität Paderborn, Warburger

Str. 100, 33095 Paderborn, Deutschland

E-Mail: michael.goller@upb.de

Lehrstuhl für Empirische Methoden der erziehungswissenschaftlichen Forschung,

Friedrich-Schiller-Universität Jena, Fürstengraben 1, 07743 Jena, Deutschland

Dr. Caroline Bonnes

Lehrstuhl für Betriebspädagogik, Fachbereich Wirtschaftswissenschaften, Universität Konstanz, Universitätsstr. 10, 78464 Konstanz, Deutschland

E-Mail: caroline.bonnes@uni-konstanz.de

Dr. Elisabeth Maué

Lehrstuhl für Wirtschaftspädagogik II, Fachbereich Wirtschaftswissenschaften, Universität Konstanz, Universitätsstr. 10, 78464 Konstanz, Deutschland

E-Mail: elisabeth.maue@uni-konstanz.de 
in signifikant positivem Zusammenhang mit resultierenden Beanspruchungsreaktionen stehen. Je transparenter, fairer und vertrauensvoller und je weniger ambivalent die Beziehung zur Seminarlehrkraft erlebt wird, desto geringer fallen die Beanspruchungsreaktionen aus. Die Ergebnisse werden hinsichtlich ihrer Relevanz für die Beziehungsarbeit im Referendariat als zentralem Ausbildungsbestandteil der zweiten Phase der Lehrer*innenausbildung diskutiert.

Schlüsselwörter Beziehung · Referendariat · Wohlbefinden · Belastung · Beanspruchung

\title{
The professional pedagogical relationship between trainee teachers and their mentors: stress factor or resource?
}

\begin{abstract}
This contribution focuses on the professional pedagogical relationship between trainee teachers and their mentors. This relationship has not yet been thoroughly investigated. The aim of the present study is to close this research gap by using a cross-sectional survey of 2583 trainee teachers and trained teachers to investigate the effects of different relationship dimensions (transparency, fairness, trust, and ambivalence) on the experienced demands and the associated stress symptoms during the teacher traineeship. The analysis was carried out using a latent moderated structural equation approach. The results show that demands caused by the core activities of teacher training as well as by colleagues has a significant positive relationship with the resulting stress symptoms. The more transparent, fair, trusting, and the less ambivalent the relationship with the mentor is experienced by the trainee teachers, the lower the resulting stress symptoms. The results are discussed regarding their relevance for the relationship work as a central training component of the second phase of teacher training.
\end{abstract}

Keywords Relationship $\cdot$ Teacher training $\cdot$ Well-being $\cdot$ Stress $\cdot$ Strain

\section{Einleitung und Zielstellung des Beitrags}

Das Referendariat galt lange Zeit als der ,,vergessene Teil der Lehrerbildung“ (Terhart 2000, S. 17). Dies hat sich mittlerweile zumindest partiell verändert: Neuere Studien nehmen beispielsweise die Bewertungspraxis in der zweiten Phase der Lehrer*innenausbildung (z.B. Kärner et al. 2018, 2019; Strietholt und Terhart 2009) oder berufliche Belastungen sowie Ressourcen angehender Lehrpersonen näher in den Blick (z.B. Beuchel und Cramer 2021; Darius et al. 2020; Klusmann et al. 2012; Košinár 2014; Menge und Schaeper 2019; Schumann 2019; Warwas et al. 2016). Hierbei zeigt sich, dass das Referendariat oft als Bewährungsprobe angesehen wird, welches Referendar*innen als ,eine Zeit voller Anpassungen, Belastungen, Ambivalenzen und Konfliktpotentiale[n]“ (Speck et al. 2007, S. 20) erleben. Dies liegt insbesondere an unterschiedlichen Belastungsfaktoren, wie beispielsweise die wahrgenommene hohe Arbeitslast, der erlebte Leistungsdruck, Konflikte mit Schüler*innen oder die als belastend empfundene Abhängigkeit von den Ausbilder*innen 
(z. B. Christ et al. 2004; Darius et al. 2020; Horstmeyer 2018; Manning und Hobson 2017; Thompson et al. 2020; Weiß et al. 2014; Wernet 2009).

Mehr und mehr rückt in diesem Zusammenhang die professionelle pädagogische Beziehung zwischen den Referendar*innen und deren Seminarlehrkräften ${ }^{1}$ in den Fokus wissenschaftlicher Untersuchungen (z. B. Horstmeyer 2018; Weiß et al. 2014). Im Allgemeinen verstehen wir darunter eine pädagogische Beziehung in einem professionellen Handlungskontext, wobei die Beziehung sowohl pädagogischer als auch professioneller Natur ist. Die Beziehung zwischen Referendar*innen und deren Seminarlehrkräften hat einerseits einen pädagogischen Charakter, da die Seminarlehrkräfte durch ihre Tätigkeit auf die berufliche Autonomie der Referendar*innen hinwirken. Hierbei lässt sich die durch einen asymmetrischen Grundcharakter geprägte Beziehung zwischen Referendar*in und Seminarlehrkraft in unterschiedlichen Spannungsfeldern verorten (siehe Abschn. 2.2.2). Zum anderen hat die Beziehung einen professionellen Charakter, da sowohl die Referendar*innen als auch die Seminarlehrkräfte jeweils der beruflichen Tätigkeit als Lehrkraft an Schulen nachgehen. Sowohl Referendar*innen als auch Seminarlehrkräfte sind hierbei gefordert, ihre gegenseitige Beziehung im Spannungsfeld zwischen wahrgenommenen Erwartungen und Erwartungserfüllung sowie zwischen eigenen Bedürfnissen und externen Anforderungen auszubalancieren (Manning und Hobson 2017; Pinnick 2020; Sembill und Kärner 2018). Die betrachtete Beziehung, welche in ihrer Grundnatur eine unterstützende und fördernde Wirkung verfolgt, kann damit sowohl eine Ressource als auch einen Belastungsfaktor darstellen (z. B. Schubarth et al. 2007). Als beanspruchend kann sich diese Beziehung insbesondere aufgrund der mit der Doppelfunktion der Seminarlehrkräfte verbundenen Rollenambiguität erweisen. Sie sollen Referendar*innen einerseits beratend unterstützen und fördern sowie eine vertrauensvolle, offene und kooperative Beziehung zu ihnen aufbauen. Andererseits treten jene jedoch häufig auch gleichzeitig als bewertende Instanzen auf und sind in die für das berufliche Fortkommen folgenreiche Beurteilung der Referendar*innen im Zuge des zweiten Staatsexamens eingebunden (z. B. Warwas et al. 2016; Wernet 2009). Die Beziehung zwischen den Referendar*innen und deren Seminarlehrkräften kann jedoch auch als Ressource bzw. Schutzfaktor dienen und zur Beanspruchungsreduktion beitragen (Drüge et al. 2014; Horstmeyer 2018; Schubarth et al. 2007). Beispielsweise verweisen Richter et al. (2011) in diesem Zusammenhang auf unterschiedliche Unterstützungsleistungen seitens der Seminarlehrkraft, welche sowohl fachlicher als auch persönlicher und sozialer Art sein können (siehe auch Pinnick 2020). Zudem zeigen Studien, dass Referendar*innen die Interaktion mit ihren Seminarlehrkräften mehrheitlich positiv erleben (Schubarth et al. 2007; Weiß et al. 2014) und dass ihnen insbesondere Hilfestellungen und Rückmeldungen helfen können, die vielfältigen Anforderungen und Belastungen des Referendariats erfolgreich zu bewältigen (Braun 2017).

Der professionellen pädagogischen Beziehung zwischen Referendar*innen und deren Seminarlehrkräften kann daher ein bedeutendes Gewicht beigemessen wer-

\footnotetext{
1 Die Bezeichnung für die Seminarlehrkraft variiert und lautet je nach Bundesland auch Ausbildungslehrkraft, Ausbildungsleiter*in, Bereichsleiter*in, Fachleiter*in, Lehrbeauftragte*r, Mentor*in oder Praktikumslehrkraft (Krüger 2014).
} 
den. Jedoch bestehen Forschungslücken zum Zusammenhang zwischen der genannten Beziehung und der Beanspruchung von Referendar*innen in der zweiten Phase der Lehrer*innenausbildung. Das Ziel der vorliegenden Studie ist es daher, anhand einer deutschlandweiten Querschnittserhebung von 2583 Referendar*innen und ausgebildeten Lehrkräften Effekte der genannten Beziehung auf die Beanspruchung im Referendariat empirisch zu untersuchen.

Nachfolgend werden zunächst relevante Begriffe und Konzepte im Zusammenhang mit der Beanspruchung im Referendariat beschrieben. Anschließend wird die professionelle pädagogische Beziehung zwischen Referendar*in und Seminarlehrkraft näher beleuchtet, angefangen bei der Struktur der Lehrer*innenausbildung und der Rolle der Seminarlehrkraft, über wesentliche Beziehungscharakteristika bis hin zur Diskussion kompensatorischer sowie verstärkender Effekte der einzelnen Beziehungsdimensionen auf die Beanspruchung im Referendariat. Die Ableitung der forschungsleitenden Fragestellung und entsprechender Untersuchungshypothesen bildet den inhaltlichen Abschluss des Theorieteils. Im empirischen Teil des Beitrags werden zunächst die Stichprobe und die Untersuchungsmethode beschrieben, bevor die Ergebnisse des latent moderierten Strukturgleichungsansatzes berichtet werden. Diese werden abschließend u. a. hinsichtlich der Beziehungsarbeit im Referendariat als zentralem Ausbildungsbestandteil diskutiert.

\section{Konzeptioneller und theoretischer Hintergrund}

\subsection{Belastung und Beanspruchung im Referendariat}

Hinsichtlich der verwendeten Begrifflichkeiten wird zunächst zwischen Belastung, Beanspruchung und Stress differenziert. Unter Belastung werden nach Semmer et al. (2006, S. 747) „,von außen, unabhängig von der Person auftretende Einflüsse, zum Beispiel Merkmale der Arbeitsaufgabe und der Arbeitsbedingungen“"verstanden, welche auch als Stressoren bezeichnet werden. Bezüglich der Kategorisierung potenzieller Belastungsquellen im beruflichen Tätigkeitsfeld von Lehrpersonen existieren verschiedene Systematiken (z. B. Rudow 2000; Van Dick 1999). Christ (2004) legt eine Systematisierung von Belastungsfaktoren speziell für das Referendariat vor (siehe auch Christ et al. 2004). Hierzu zählen insbesondere organisatorische und strukturelle Faktoren (z.B. zu große Klassen, zusätzliche Verwaltungsarbeit, Vermischung von Privat- und Berufsleben), Merkmale und Verhaltensweisen der Schüler*innen (z.B. Disziplinprobleme, Heterogenität, Konflikte), ausbildungsspezifische Faktoren (z. B. Unterrichtsbesuche, Unterrichtsvor- und -nachbereitung) aber auch die Beziehung zu Kolleg*innen (z.B. Konflikte, Abspracheprobleme) (hierzu auch Kärner et al. 2021a).

Zur Modellierung von Beanspruchung im Lehrberuf existieren unterschiedliche Ansätze (im Überblick Van Dick und Stegmann 2013), wobei denjenigen Modellen, welche im Kern auf den Ideen des transaktionalen Stressmodells nach Lazarus (1966) basieren (z. B. Kyriacou 2001; Kyriacou und Sutcliffe 1978; Rudow 1994; Van Dick 1999), aufgrund ihres weitreichenden Erklärungspotenzials eine zentrale Bedeutung zukommt. Im Zentrum dieser Modelle steht die Annahme, dass Bean- 
spruchung durch die subjektive Bewertung potenzieller Belastungsquellen entsteht, welche grundsätzlich als irrelevant, als herausfordernd oder als bedrohlich bewertet werden können. Wird eine Belastungsquelle als Bedrohung bewertet und übersteigt die damit verbundene wahrgenommene Beanspruchung die personen- und/ oder umweltbezogenen Ressourcen oder werden diese bezogen auf eine angemessene Bewältigung als defizitär eingeschätzt, wird Beanspruchung zu Stress (Sembill 2012; Semmer et al. 2006). Entsprechende Beanspruchungsreaktionen können sich bei (angehenden) Lehrpersonen in psychischen (z.B. emotionale Erschöpfung, geringes Wohlbefinden) oder somatischen (z. B. Kopfschmerzen, Übelkeit) Symptomen äußern (Gardner 2010; Harmsen et al. 2018; Lazarus 1966; Lazarus und Folkman 1984). Klusmann et al. (2012) fanden in diesem Zusammenhang heraus, dass die emotionale Erschöpfung, welche von angehenden Lehrpersonen berichtet wird, im Durchschnitt mit derjenigen von Lehrpersonen vergleichbar ist, welche ihren Beruf bereits mehrere Jahre ausüben. Wie die Studie von Christ et al. (2004) zeigt, sind insbesondere unterrichts- und ausbildungsbezogene Belastungsfaktoren im Vorbereitungsdienst mit einem geringeren psychischen Wohlbefinden und vermehrten körperlichen Beschwerden verbunden. Geschlechtsspezifische Unterschiede werden von Chaplain (2008) berichtet, wobei Lehramtsanwärterinnen im Vergleich zu Lehramtsanwärtern eine höhere psychische Beanspruchung erleben.

Im Hinblick auf das Job Demands-Resources Modell können Ressourcen bzw. Schutzfaktoren als Moderatoren fungieren, da sie einen Einfluss auf die Beziehung zwischen Belastungen und resultierenden Beanspruchungsreaktionen haben und als Puffer wirken können (Affolter 2019; Demerouti et al. 2001; Xanthopoulou et al. 2007). Es kann also davon ausgegangen werden, dass objektiv gleiche Belastungssituationen je nach emotionaler Bewertung und abhängig von individuellen Ressourcen als unterschiedlich beanspruchend empfunden werden (Sembill 2012).

\subsection{Die professionelle pädagogische Beziehung zwischen Referendar*in und Seminarlehrkraft}

\subsubsection{Struktur der Lehrer*innenausbildung und Rolle der Seminarlehrkraft}

Die Struktur der Lehrer*innenausbildung in Deutschland gliedert sich in zwei Grundphasen. Die erste Phase findet an der Hochschule statt und schließt mit dem ersten Staatsexamen bzw. dem Master ab. Das Referendariat stellt die zweite, berufspraktische Phase dar und gilt mit dem Erreichen des zweiten Staatsexamens als abgeschlossen (Bellenberg und Thierack 2003; Krüger 2014; Strietholt und Terhart 2009; Terhart 2000). Das übergeordnete Ziel des Referendariats besteht darin, die in der ersten Phase erworbenen Kenntnisse, Fertigkeiten und Erfahrungen zu vertiefen und zu erweitern sowie die Handlungsfähigkeit in den Kompetenzbereichen zu erlangen, welche in den von der Kultusministerkonferenz (2018) verabschiedeten Standards für die Lehrer*innenausbildung festgeschrieben sind (Bellenberg und Thierack 2003; Krüger 2014; Schubarth et al. 2007; Schulte 2008).

Das Referendariat findet in der Regel an zwei Lernorten statt: am Studienseminar und an einer Ausbildungsschule (Bellenberg und Thierack 2003; Schulte 2008; Terhart 2000). In der Ausbildungsschule stehen unterrichtliches, erzieherisches, be- 
ratendes sowie beurteilendes Handeln im Fokus, was durch Hospitationen sowie eigenverantwortlichen Unterricht erfolgt (Schubarth et al. 2007; Terhart 2000). Die Ausbildung am Studienseminar beinhaltet im Wesentlichen pädagogische und fachbezogene Seminarveranstaltungen sowie Unterrichtsbesuche und Lehrproben (Braun 2017; Krüger 2014; Munderloh 2018). Die Seminarlehrkräfte sind hierbei in die Ausbildungs-, Beratungs- und Beurteilungsprozesse am Studienseminar involviert. Im Allgemeinen umfasst das Tätigkeitsgebiet der Seminarlehrkräfte die fachliche, didaktische sowie pädagogische Ausbildung der Referendar*innen (Krüger 2014; Kruse-Moosmayer und Zoller 2007). Im Detail beinhaltet dies die Leitung fachdidaktischer und pädagogischer Seminare, den Besuch von Referendar*innen im Unterricht, die Betreuung und Beratung der Referendar*innen hinsichtlich fachlicher Fragen, die Beobachtung und Unterstützung des Professionalisierungsprozesses, das Führen von Ausbildungsgesprächen, die Zusammenarbeit mit Ausbildungsschulen sowie die Beteiligung an der zweiten Staatsprüfung (Krüger 2014). Hierbei wird die Doppelrolle der Seminarlehrkräfte deutlich: Einerseits müssen sie betreuende sowie beratende Funktionen wahrnehmen und andererseits sind sie implizit oder explizit in die notenwirksame Beurteilung der angehenden Lehrkräfte im Rahmen des zweiten Staatsexamens involviert (Hintz 2000; Lenhard 2004; Munderloh 2018; Schubarth et al. 2007; Warwas et al. 2016; Weiß et al. 2014; Wernet 2009).

\subsubsection{Charakteristika der professionellen pädagogischen Beziehung zwischen Referendar*in und Seminarlehrkraft und deren Wirkungen als Ressource oder Belastungsfaktor}

Den Seminarlehrkräften wird innerhalb des Referendariats hinsichtlich der fachlichen und persönlichen Entwicklung der Referendar*innen eine große Bedeutung beigemessen (Bölting und Thomas 2007; Krüger 2014). Zum einen zeigen Studien die protektiven Effekte einer als positiv erlebten Beziehung zwischen Seminarlehrkräften und Referendar*innen hinsichtlich ihren Beanspruchungsreaktionen (z.B. Braun 2017; Horstmeyer 2018; Richter et al. 2011; Warwas et al. 2016). Zum anderen kann die Beziehung zwischen Seminarlehrkraft und Referendar*in, welche systembedingt durch Asymmetrie, starke Hierarchien, Unfreiwilligkeit und zeitliche Befristung gekennzeichnet ist (Košinár 2013; Looser 2019; Munderloh 2018; Pinnick 2020; Schweer 2008; Weiß et al. 2014), die Wirkung potenzieller Belastungsquellen und -situationen zusätzlich verstärken bzw. selbst zum Belastungsfaktor werden.

Gemäß Helsper (2004, S. 63) ist der „Bezugspunkt der professionellen und insbesondere der pädagogischen Tätigkeit [...] die Autonomie der Lebenspraxis, die entweder noch nicht entfaltet, vorübergehend beeinträchtigt oder längerfristig erschwert bzw. blockiert ist“. Übertragen auf das Referendariat kann der Bezugspunkt des Handelns der Seminarlehrkräfte in der Ermöglichung und Stärkung der beruflichen Autonomie der Referendar*innen gesehen werden. Die für die Lehrprofessionalität konstitutiven Antinomien (Helsper 2004) lassen sich vor dem Hintergrund der professionellen pädagogischen Beziehung zwischen Referendar*innen und deren Seminarlehrkräften diskutieren. So ist diese Beziehung durch das Spannungsfeld von Nähe und Distanz (Näheantinomie) gekennzeichnet. Darüber hinaus ist die Un- 
terstellung und kontinuierliche Herstellung einer Vertrauensbasis unter besonderen, nicht unbedingt vertrauensförderlichen Rahmenbedingungen erforderlich (Vertrauensantinomie). Die Symmetrie- bzw. Machtantinomie ergibt sich aus der überlegenen Position der Seminarlehrkräfte hinsichtlich Wissen und Erfahrungen, aber auch deren Macht, etwa durch deren Beteiligung an der Benotung. An diesem Punkt wird eine weitere Parallele zwischen dem Referendariat als Lern- und Ausbildungsphase und der Schule als Lernort deutlich: Der für die schulische Bewertung konstitutive „Widerstreit von Wahrheit, Wahrhaftigkeit, Gerechtigkeit und Fürsorge“ (Helsper 2004, S. 50) lässt sich nicht nur auf die Benotung der Referendar*innen durch ihre Seminarlehrkräfte übertragen, sondern stellt aufgrund der besonderen Konstellation, dass Seminarlehrkräfte gleichzeitig Kolleg*innen der Referendar*innen sind, eine Verschärfung dar. Die Gleichzeitigkeit von asymmetrischer und symmetrischer, kollegialer Beziehung verweist auf einen Zusammenhang mit der Autonomieantinomie, bei der die Ermöglichung von Autonomie (etwa Eigenständigkeit bei der Unterrichtsvorbereitung, -durchführung und -nachbereitung) durch die Regularien für das Referendariat begrenzt wird. Die benannten Antinomien sowie die bei Bewertungen auftretenden Spannungsfelder (Helsper 2004) bilden einen übergeordneten inhaltlichen Rahmen für die Beschreibung der professionellen pädagogischen Beziehung zwischen Referendar*innen und deren Seminarlehrkräften. In Orientierung an den beschriebenen Antinomien professioneller pädagogischer Beziehungen charakterisieren und operationalisieren Kärner et al. (2021b) die folgenden vier Facetten der betrachteten Beziehung, die sich sowohl kompensatorisch als auch verstärkend auf das Belastungserleben im Referendariat auswirken können: Transparenz, Fairness, Vertrauen sowie Ambivalenz (z. B. Bauer 2006; Helsper und Hummerich 2014; Helsper und Wiezorek 2006; Heymann 2009; Krautz und Schieren 2013; Munderloh 2018; Prengel 2019; Schweer 1996; Sembill 1992; Weiß et al. 2014):

1. Die professionelle pädagogische Beziehung zwischen Referendar*innen und deren Seminarlehrkräften lässt sich durch die Ausprägung auf der Dimension Transparenz charakterisieren. Die Beziehungsqualität und damit verbunden auch die Betreuungsqualität werden entscheidend dadurch bestimmt, ob Offenheit für Fragen und Kritik besteht und ob insbesondere die Leistungsbewertung durch die Seminarlehrkraft als nachvollziehbar wahrgenommen wird (Abs et al. 2009; Leshem 2012; Manning und Hobson 2017; Munderloh 2018; Pinnick 2020; Warwas et al. 2016). Grundlegend hierfür sind verbindliche, klar, verständlich und nachvollziehbar kommunizierte Leistungserwartungen und Bewertungskriterien, welche eine wesentliche Basis der fachlichen Unterstützung darstellen (Kärner et al. 2018; Zsargo und Palmer 2019). In ihrer Studie konnten Kärner et al. (2019) zeigen, dass transparente und offen kommunizierte Leistungserwartungen und Bewertungskriterien sowie lernförderliche Rückmeldungen zu den erbrachten Leistungen mit einer höheren Zufriedenheit und einem geringeren Beanspruchungserleben im Referendariat einhergehen. In dem Fall kann die Dimension Transparenz bei einer hohen Ausprägung als Ressource in Bezug auf die Belastungen im Referendariat postuliert werden (siehe auch Horstmeyer 2018). Nehmen Referendar*innen jedoch die Kriterien einer transparenten Leistungsbewertung seitens der 
Seminarlehrkräfte nicht als erfüllt wahr, erleben sie den Bewertungskontext häufig als ungerecht und willkürlich (Munderloh 2018; Schubarth et al. 2007).

2. Die im Rahmen der professionellen pädagogischen Beziehung erlebte Fairness ist ebenfalls von zentraler Bedeutung. So ist aus der Forschung zu Lehrer*innenSchüler*innen-Beziehungen bekannt, dass Schüler*innen Gerechtigkeit als eine der zentralen Eigenschaften nennen, die sie sich von ihrer Lehrperson wünschen (Donat et al. 2017; Hofer et al. 1986) und dass es den meisten Lehrpersonen selbst wichtig ist, fair, gerecht und respektvoll gegenüber ihren Schüler*innen zu agieren (Donat et al. 2017; Kanders 2000; Schweer et al. 2017). Ausbildungs- und Bewertungsprozesse im Referendariat dürften seitens der Referendar*innen als fair erlebt werden, wenn sie an Entscheidungen ihre Ausbildung betreffend partizipieren können, sie den Eindruck haben, dass die von ihnen erbrachten Leistungen angemessen honoriert werden und sie sich mit Würde und Respekt behandelt fühlen (z.B. Colquitt 2001; Heid 1992; Maier et al. 2007). Als ungerechtfertigt erlebte Kritik seitens der Seminarlehrkräfte wird hingegen von den Referendar*innen als irritierend oder verletzend und damit als beanspruchend wahrgenommen (Bovet und Frommer 2001). Damit kann ein hoher Grad an erlebter Fairness als Ressource in Bezug auf die Belastung im Referendariat angesehen werden.

3. Ein weiteres Merkmal professioneller pädagogischer Beziehungen stellt Vertrauen dar. Bovet und Frommer (2001; ähnlich Kentzer et al. 2019; Leshem 2012) beschreiben im Kontext der Ausbildung von Lehrpersonen den Aufbau einer vertrauensvollen Beziehung zwischen Referendar*in und Seminarlehrkraft als notwendige Basis für einen gelungenen Beratungsprozess, welcher durch Authentizität, gegenseitige Wertschätzung und Empathie geprägt ist. Da die interpersonale Situation zwischen Seminarlehrkraft und Referendar*in aufgrund der hierarchischen Ausbildungsstruktur faktisch durch ein formales Machtgefälle gekennzeichnet ist und das Risiko zu vertrauen für die rangniedrigere Person (d.h. der*die Referendar*in) in der Regel höher ist, muss die Seminarlehrkraft Vertrauensvorleistungen erbringen, um den Prozess der Vertrauensbildung in Gang zu setzen (Heinrich 2011; Jones und Pittman 1982; Schweer 1996, 2012; Wernet 2009). Empirisch zeigt sich beispielsweise, dass das wahrgenommene Vertrauen in pädagogischen Beziehungen mit einer höheren Angstfreiheit der Lernenden in Zusammenhang steht (Schweer 2008). Problematische und damit belastende pädagogische Beziehungen sind insbesondere durch geringes Vertrauen und einem damit einhergehenden Abhängigkeitserleben sowie mangelnde Reziprozität gekennzeichnet (z.B. Ferguson 2017; Kaststaller et al. 2014; Kiper 2014; Laireiter und Lettner 1993; Leshem 2012; Wolf 1998). Da „Vertrauen eine soziale und personale Ressource“ (Schweer et al. 2017, S. 523) darstellt, dürfte sich ein hohes Maß an Vertrauen positiv im Sinne einer Ressource auf die Beanspruchung im Referendariat auswirken.

4. Aufgrund der funktionsbedingten Doppelrolle der Seminarlehrkräfte und der damit verbundenen Rollenambiguität stellt die erlebte Ambivalenz im Sinne einer Doppelgerichtetheit (Wirtz und Strohmer 2017) einen weiteren zentralen Bestandteil der differenzierten Beziehungsanalyse (Prengel 2019) zwischen Referendar*in und Seminarlehrkraft dar. Kennzeichen ambivalenter Beziehungen sind die erlebte Inkompatibilität unterschiedlicher Gefühle und/oder Kognitionen, die Simultani- 
tät dieser Gefühle bzw. Kognitionen sowie eine evaluative Komponente, d.h. eine Valenz-Bewertung im Sinne von ,gut-schlecht“ oder ,positiv-negativ“ (Ziegler 2010). Ambivalente Beziehungskonstellationen können aus sowohl verletzenden als auch anerkennenden Interaktionen mit ein und dem*derselben Interaktionspartner*in resultieren (Prengel 2019). Insbesondere die Doppelfunktion der Seminarlehrkraft kann sich dann belastend auf die Beziehung auswirken, wenn die jeweiligen Rollen (Berater*in vs. Beurteiler*in) nicht angemessen voneinander getrennt werden bzw. sich hinsichtlich des jeweiligen Rollenverhaltens inkonsistente Verhaltensweisen zeigen (Manning und Hobson 2017; Pinnick 2020; Wernet 2009; Zsargo und Palmer 2019). Die Referendar*innen müssen sich darauf verlassen können, dass die Seminarlehrkraft zwischen Ausbildungssituationen und Beurteilungssituationen trennen kann und es beispielsweise nicht zu ungerechtfertigten bzw. unangekündigten Bewertungssituationen kommt (Schubarth et al. 2007). Fehlt diese Trennung oder wird sie nicht konsistent eingehalten und offen kommuniziert, kann dies aufgrund der systembedingten ungleichen Machtverteilung und der damit verbundenen asymmetrischen Beziehungsstruktur wiederum den Aufbau einer vertrauensvollen Beziehung hemmen bzw. verhindern (Wernet 2009). Damit die rollenbedingte Ambivalenz keine (zusätzliche) Belastung im Referendariat darstellt, müssen sich die Seminarlehrkräfte in ihrer Funktionsausübung geschickt im Spannungsfeld zwischen Beratung und Beurteilung bewegen (Hintz 2000; Lenhard 2004; Munderloh 2018; Schubarth et al. 2007; Weiß et al. 2014; Wernet 2009).

Zusammenfassend lässt sich festhalten, dass die professionelle pädagogische Beziehung zwischen Referendar*innen und ihren Seminarlehrkräften durch die vier Beziehungsfacetten Transparenz, Fairness, Vertrauen und Ambivalenz vor dem Hintergrund der von Helsper (2004) beschriebenen Antinomien pädagogischen Handelns charakterisiert werden kann. Hervorzuheben bleibt, dass die vier Beziehungsfacetten miteinander in Zusammenhang stehen, d.h., nicht unabhängig voneinander sind. Darüber hinaus kann konstatiert werden, dass hohe Ausprägungen bei Transparenz, Fairness und Vertrauen sowie eine geringe Ausprägung der erlebten Ambivalenz tendenziell mit einer positiven Valenz besetzt sind. Geringe Ausprägungen bei Transparenz, Fairness und Vertrauen sowie eine hohe Ausprägung der erlebten Ambivalenz sind tendenziell mit einer negativen Valenz verbunden.

\subsection{Fragestellung und Hypothesen}

Bezugnehmend auf die vorangehenden Ausführungen ist zum einen davon auszugehen, dass Belastungen im Referendariat je nach individueller Bewertung als unterschiedlich beanspruchend erlebt werden können (z. B. Kyriacou 2001; Lazarus 1966; Van Dick und Stegmann 2013). Zum anderen können insbesondere beziehungsbezogene Merkmale als Ressource fungieren, sofern diese positiv konnotiert sind, oder aber als zusätzlicher Belastungsfaktor, sofern diese negativ konnotiert sind, und damit entsprechende Einflüsse auf die resultierenden Beanspruchungsreaktionen aufweisen (z. B. Bovet und Frommer 2001; Kärner et al. 2019; Weiß et al. 2014; Wernet 2009). Weiterhin ist anzunehmen, dass beziehungsbezogene Merkma- 
le, je nach Konnotierung, Einfluss auf den Zusammenhang zwischen potenziellen Belastungsquellen und resultierenden Beanspruchungsreaktionen haben (Demerouti et al. 2001; Xanthopoulou et al. 2007).

Vor diesem Hintergrund wird der Fragestellung nachgegangen, inwieweit sich die professionelle pädagogische Beziehung zwischen Referendar*innen und deren Seminarlehrkräften auf die Beanspruchung im Referendariat auswirkt. Basierend auf den bisherigen Ausführungen werden folgende Untersuchungshypothesen aufgestellt:
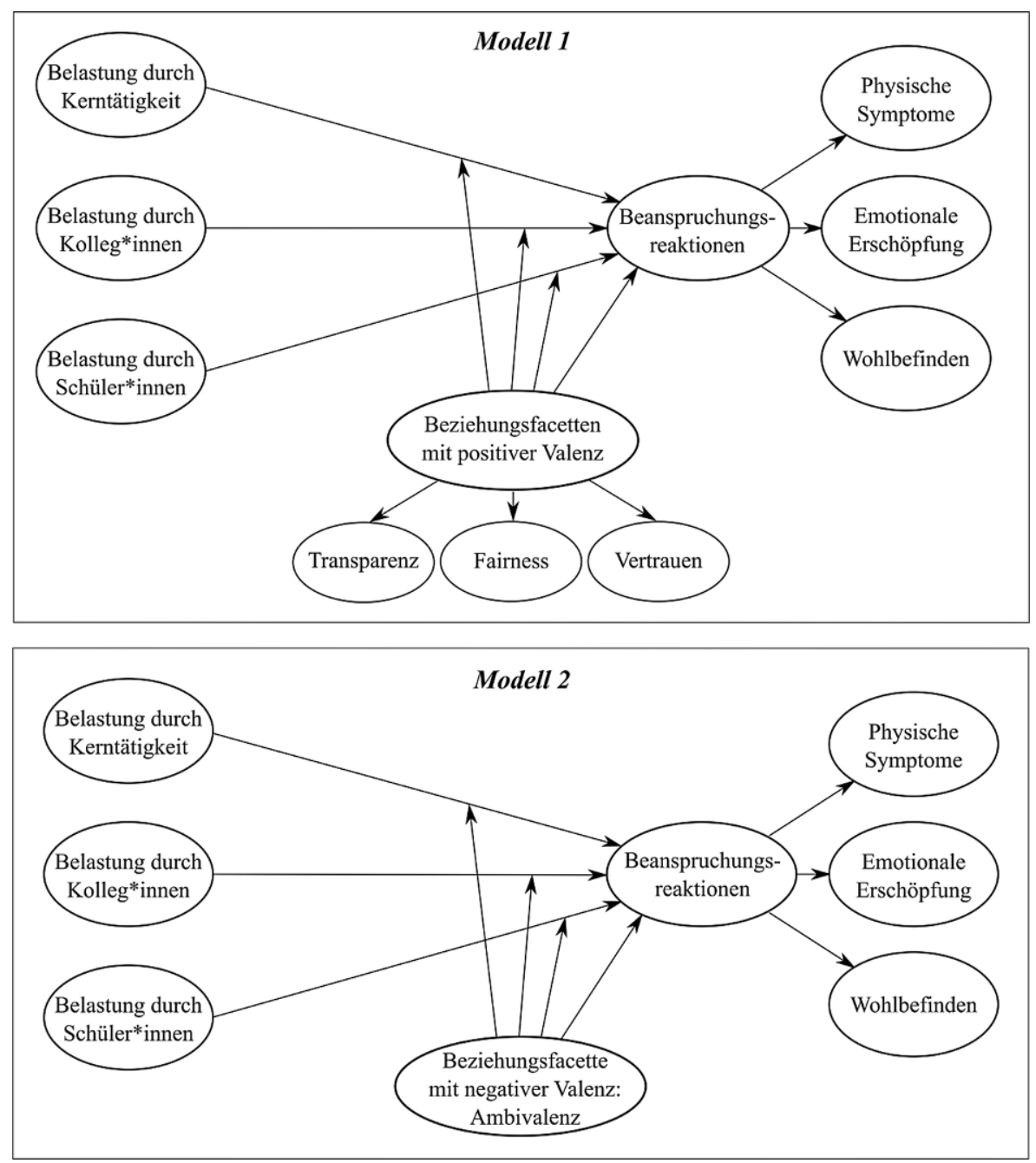

Abb. 1 Analysemodelle 
(H1) Je belastender und bedrohlicher die beruflichen Belastungsquellen erlebt werden, desto stärker fallen die Beanspruchungsreaktionen aus (Haupteffekte der Belastungsquellen; siehe Abschn. 2.1).

(H2) Je transparenter, fairer und vertrauensvoller und je weniger ambivalent die Beziehung zur Seminarlehrkraft seitens der Referendar*innen erlebt wird, desto schwächer fallen die Beanspruchungsreaktionen aus (Haupteffekte der Beziehungsfacetten; siehe Abschn. 2.2.2).

(H3) Je transparenter, fairer und vertrauensvoller und je weniger ambivalent die Beziehung zur Seminarlehrkraft seitens der Referendar*innen erlebt wird, desto geringer fällt der Effekt der Belastungsquellen auf die Beanspruchungsreaktionen aus (Interaktionseffekte zwischen den Beziehungsfacetten und den Belastungsquellen; siehe Abschn. 2.2.2).

Abb. 1 stellt die angenommenen Zusammenhänge grafisch dar (die Bezeichnungen „Modell 1“" sowie „Modell 2" beziehen sich auf das Auswertungsvorgehen, siehe Abschn. 3.3 und 4.2).

\section{Methode}

\subsection{Untersuchungsmethode und Stichprobe}

Zur Überprüfung der Hypothesen wurde eine deutschlandweite Querschnittserhebung von Referendar*innen sowie Lehrkräften mit zweitem Staatsexamen durchgeführt. Zur Stichprobenakquise wurde die Studie im März 2020 in verschiedenen sozialen Netzwerken (z.B. Facebook, Twitter, 4teachers.de, Xing), Verteilern der Gewerkschaft für Erziehung und Wissenschaft (GEW) sowie im direkten Umfeld der Autor*innen bekannt gemacht und beworben. An der onlinebasierten Befragung nahmen 3033 Personen teil. Insgesamt wiesen 316 Fälle bei mehr als 30\% der Variablen fehlende Werte auf und wurden daher aus der Stichprobe entfernt. Darüber hinaus wurden 134 Befragte, deren Referendariat zum Zeitpunkt der Befragung länger als fünf Jahre zurücklag, aus der Analyse ausgeschlossen, da Erinnerungseffekte die Ergebnisse verzerren könnten.

Auswertbare Daten liegen somit von 2583 gültigen Fällen vor $(81,6 \%$ weiblich). Im Durchschnitt waren die befragten Personen 29,0 Jahre $(S D=4,01)$ alt. Die Verteilung der Proband*innen im Hinblick auf Bundesland und Schulart getrennt nach Referendar*innen und Lehrkräften mit zweitem Staatsexamen ist in Tab. 1 dargestellt. Alle nachfolgenden Analysen werden separat für die Gruppen der Referendar*innen (Subskript: R) und der Lehrkräfte mit zweitem Staatsexamen (kurz: Lehrkräfte, Subskript: L) durchgeführt. Hiervon wird sich einerseits versprochen, weitere potenzielle Erinnerungseffekte bei den bereits ausgebildeten Lehrkräften zu identifizieren und somit die Ergebnisse der Studie nicht zu verzerren. Andererseits ermöglicht das Vorgehen, die Robustheit der Befunde durch eine Replikation der Analysen in zwei unabhängigen Teilstichproben zu überprüfen. 
Tab. 1 Stichprobenbeschreibung

\begin{tabular}{|c|c|c|}
\hline Merkmal & Referendar*innen & $\begin{array}{l}\text { Lehrkräfte mit } \\
\text { 2. Staatsexamen }\end{array}$ \\
\hline \multicolumn{3}{|l|}{ Bundesländer } \\
\hline Baden-Württemberg & 161 & 102 \\
\hline Bayern & 366 & 152 \\
\hline Berlin & 9 & 13 \\
\hline Brandenburg & 5 & 5 \\
\hline Bremen & 0 & 2 \\
\hline Hamburg & 24 & 12 \\
\hline Hessen & 84 & 29 \\
\hline Mecklenburg-Vorpommern & 13 & 0 \\
\hline Niedersachsen & 92 & 55 \\
\hline Nordrhein-Westfalen & 807 & 376 \\
\hline Rheinland-Pfalz & 41 & 21 \\
\hline Saarland & 2 & 3 \\
\hline Sachsen & 56 & 19 \\
\hline Sachsen-Anhalt & 14 & 7 \\
\hline Schleswig-Holstein & 27 & 13 \\
\hline Thüringen & 54 & 17 \\
\hline \multicolumn{3}{|l|}{ Schulart (Mehrfachauswahl möglich) } \\
\hline Grundschule & 402 & 153 \\
\hline Hauptschule/Mittelschule & 92 & 28 \\
\hline Realschule & 137 & 84 \\
\hline Gemeinschaftsschule & 16 & 7 \\
\hline Gesamtschule & 153 & 72 \\
\hline (Integrierte) Gesamtschule mit Gymnasialer Oberstufe & 17 & 5 \\
\hline (Integrierte) Sekundarschule/Oberschule/Stadtteilschule & 20 & 9 \\
\hline Gymnasium & 649 & 344 \\
\hline Berufliche Schule & 167 & 72 \\
\hline Förderschule & 150 & 71 \\
\hline Sonstige & 3 & 4 \\
\hline \multicolumn{3}{|l|}{ Zeitpunkt im Referendariat } \\
\hline 1. Jahr bzw. Halbjahr (wenn Referendariat $\leq 18$ Monate) & 754 & NA \\
\hline 2. Jahr & 893 & NA \\
\hline Wiederholer*in & 24 & NA \\
\hline Sonstige & 85 & NA \\
\hline \multicolumn{3}{|l|}{ Jahre seit Abschluss des Referendariats } \\
\hline$<1 \mathrm{Jahr}$ & NA & 98 \\
\hline 1-2 Jahr(e) & NA & 527 \\
\hline 3-4 Jahre & NA & 129 \\
\hline 5 Jahre & NA & 41 \\
\hline Summe ${ }^{\text {a }}$ & 1756 & 826 \\
\hline
\end{tabular}

Anmerkungen: $N A=$ nicht anwendbar; Sollten sich die Einzelkategorien nicht zur Gesamtsumme der Befragten in den (Teil-)Stichproben addieren, dann liegt dies an fehlenden Werten.

${ }^{\text {a }}$ Eine Person gab nicht an, ob sie sich im Referendariat befindet oder das zweite Staatsexamen abgeschlossen hat 


\subsection{Operationalisierung der Variablen}

\subsubsection{Belastungsquellen und deren subjektive Bewertung}

Nach Sichtung vorliegender Systematisierungen potenzieller Belastungsquellen im Lehrberuf bzw. Referendariat (siehe Abschn. 2.1) unterscheidet der vorliegende Beitrag Belastungen aufgrund der Kerntätigkeit im Rahmen des Referendariats (z.B. Workload bzgl. Unterrichtsvor- und -nachbereitung), aufgrund des Umgangs mit Schüler*innen (z.B. Disziplinprobleme seitens der Schüler*innen und daraus resultierende Konflikte) sowie aufgrund der Zusammenarbeit mit Kolleg*innen (z.B. Konflikte im Kollegium). Die jeweiligen Items zur Erfassung der Belastungsquellen im Referendariat lehnen sich an existierende Erfassungssystematiken ${ }^{2}$ an (Christ 2004; Christ et al. 2004; Rudow 2000; Van Dick 1999). Zusätzlich wurden eigene Items entwickelt.

Zum einen wurde für jedes Item gefragt, wie belastend die Befragten die betreffenden Aspekte während des Referendariats empfinden bzw. empfanden (4-stufige Likert-Skala von $1=$, ,̈̈berhaupt nicht belastend“ bis $4=$,,sehr belastend“). Zum anderen wurde für jedes Item gefragt, wie persönlich relevant die Befragten die Belastungsquellen einschätzen ( $1=$,irrelevant", $2=$,herausfordernd“", $3=$ =, bedrohlich“"). Jedes der Itempaare (Belastungseinschätzung und Relevanzeinschätzung) wurde im Anschluss miteinander multipliziert, sodass für jede Aussage ein Gesamtscore aus Belastung und Relevanz resultierte ( $1=$, überhaupt nicht belastend und irrelevant“ bis $12=$,sehr belastend und bedrohlich“). Diese Vorgehensweise orientiert sich an der Grundidee des transaktionalen Stressmodells, nach welchem eine Belastungsquelle erst dann Beanspruchungsreaktionen und letztendlich Stress nach sich zieht, wenn der betreffenden Belastungsquelle eine entsprechende Relevanz attribuiert wird (siehe Abschn. 2.1). Tab. 2 beinhaltet nähere Informationen zu den erfassten Belas-

Tab. 2 Operationalisierung der Belastungsquellen

\begin{tabular}{lllcc}
\hline Faktor & $\begin{array}{l}\text { Anzahl } \\
\text { Items }\end{array}$ & Beispielitem & $\omega_{\mathrm{R}}$ & $\omega_{\mathrm{L}}$ \\
\hline $\begin{array}{l}\text { Belastung durch } \\
\text { Kerntätigkeit }\end{array}$ & 8 & $\begin{array}{l}\text { Die Unterrichtsvor- und -nachbereitung } \\
\text { empfinde ich als ... }\end{array}$ & 0,79 & 0,80 \\
$\begin{array}{l}\text { Belastung durch } \\
\text { Schüler*innen }\end{array}$ & 4 & $\begin{array}{l}\text { Das Verhalten der Schüler*innen im } \\
\text { Unterricht empfinde ich als ... }\end{array}$ & 0,74 & 0,73 \\
$\begin{array}{l}\text { Belastung durch } \\
\text { Kolleg*innen }\end{array}$ & 3 & $\begin{array}{l}\text { Den Umgang mit Kolleg*innen empfinde } \\
\text { ich als ... }\end{array}$ & 0,62 & 0,65 \\
\hline
\end{tabular}

Anmerkungen: $\omega_{\mathrm{R}}=$ Kongenerische Reliabilität der Referendar*innen, $\omega_{\mathrm{L}}=$ Kongenerische Reliabilität der Lehrkräfte mit zweitem Staatsexamen; Belastungseinschätzung auf 4-stufiger Likert-Skala von $1=$,überhaupt nicht belastend“ bis $4=$,,sehr belastend“; Relevanzeinschätzung auf 3-stufiger Likert-Skala: 1= ,,irrelevant“, 2 =, ,herausfordernd“", 3= ,bedrohlich“; Abfrage für die Lehrkräfte mit 2. Staatsexamen in Vergangenheitsform. Adaptation der Items nach Christ (2004), Christ et al. (2004), Rudow (2000) und Van Dick (1999).

\footnotetext{
2 Wir referenzieren hierbei zum einen auf Kategorisierungen, welche sich auf allgemeine Belastungsfaktoren im Lehrberuf beziehen (z. B. Umgang mit Kolleg*innen), da diese auch für Referendar*innen gelten. Zum anderen beziehen wir uns auf Belastungsfaktoren, welche für Referendar*innen aufgrund ihrer Ausbildungssituation spezifisch sind (z. B. Vorbereitung auf Lehrproben).
} 
tungsquellen einschließlich der Anzahl der Items, eines Beispielitems und $\omega$ als Maß für die kongenerische Reliabilität der Skalen (in Korrespondenz zu Cronbachs Alpha mindestens $>0,60$ oder besser $>0,70$ ).

\subsubsection{Merkmale der Beziehung zwischen Seminarlehrkraft und Referendar*in}

Die vier Facetten der Beziehungsqualität Transparenz, Fairness, Vertrauen sowie Ambivalenz wurden mittels eines Instruments von Kärner et al. (2021b) operationalisiert (siehe Tab. 3). Die Items wurden auf einer 6-stufigen Likert-Skala von $1=$,trifft nicht zu“ bis $6=$,trifft voll zu“ abgefragt. Aufgrund bundeslandspezifischer Variationen in der Bezeichnung der Rolle der Seminarkraft sowie teils variierender Aufgabenbereiche der betreffenden Personen erfolgte einleitend eine Klärung des Begriffs „Seminarlehrkraft“, um im Zuge der Befragung ein möglichst einheitliches Verständnis bezüglich des betreffenden Personenkreises sicherzustellen.

\subsubsection{Beanspruchungsreaktionen}

Zur Erfassung der Beanspruchungsreaktionen sollten die Befragten angeben, wie häufig das jeweils genannte physische oder psychische Symptom bzw. Erleben während ihres Referendariats auftritt bzw. auftrat. Die Items zur physischen Symptomatik sowie zum Wohlbefinden wurden von Lohaus et al. (2018) ${ }^{3}$ adaptiert, die Items zur emotionalen Erschöpfung von Maslach und Jackson (1981). Alle Items wurden auf einer 5-stufigen Skala von 1=,,nie“ bis 5=,,sehr oft“" abgefragt (siehe Tab. 4).

Tab. 3 Operationalisierung der Beziehungsmerkmale

\begin{tabular}{|c|c|c|c|c|}
\hline Variablen & $\begin{array}{l}\text { Anzahl } \\
\text { Items }\end{array}$ & Beispielitem & $\omega_{\mathrm{R}}$ & $\omega_{\mathrm{L}}$ \\
\hline Transparenz & 4 & $\begin{array}{l}\text { Meine Seminarlehrkraft kommuniziert klar, welche } \\
\text { Kompetenzen ich beherrschen muss. }\end{array}$ & 0,86 & 0,89 \\
\hline Fairness & 4 & Meine Seminarlehrkraft behandelt mich fair. & 0,87 & 0,90 \\
\hline Vertrauen & 4 & $\begin{array}{l}\text { Vor meiner Seminarlehrkraft kann ich zugeben, dass ich } \\
\text { etwas nicht verstanden habe. }\end{array}$ & 0,85 & 0,87 \\
\hline Ambivalenz & 4 & $\begin{array}{l}\text { Ich freue mich, wenn meine Seminarlehrkraft mich } \\
\text { unterstützt, aber gleichzeitig ärgere ich mich darüber, } \\
\text { dass ich von ihr abhängig bin. }\end{array}$ & 0,64 & 0,64 \\
\hline
\end{tabular}

Anmerkungen $: \omega_{\mathrm{R}}=$ Kongenerische Reliabilität der Referendar*innen, $\omega_{\mathrm{L}}=$ Kongenerische Reliabilität der Lehrkräfte mit zweitem Staatsexamen; 6-stufige Likert-Skala von $1=$,trifft nicht zu“ bis $6=$,,trifft voll zu“; Abfrage für die Lehrkräfte mit 2. Staatsexamen in Vergangenheitsform. Items entnommen aus Kärner et al. (2021b)

\footnotetext{
3 Wenngleich der Fragebogen von Lohaus et al. (2018) zur Erfassung von Stress und Stressbewältigung im Kindes- und Jugendalter entwickelt wurde, sind viele Items so allgemein formuliert, dass sie auch bei Erwachsenen abgefragt werden können. Wenn dies bei einem Item nicht der Fall war, haben wir es entsprechend an den Kontext von Erwachsenen angepasst.
} 
Tab. 4 Operationalisierung der Beanspruchungsreaktionen

\begin{tabular}{|c|c|c|c|c|}
\hline Variablen & $\begin{array}{l}\text { Anzahl } \\
\text { Items }\end{array}$ & Beispielitem & $\omega_{\mathrm{R}}$ & $\omega_{\mathrm{L}}$ \\
\hline $\begin{array}{l}\text { Physische Sympto- } \\
\text { me }\end{array}$ & 6 & [Im Referendariat] ... habe ich Bauchweh. & 0,82 & 0,82 \\
\hline $\begin{array}{l}\text { Emotionale Er- } \\
\text { schöpfung }\end{array}$ & 4 & $\begin{array}{l}\text {... fühle ich mich von meiner Arbeit emo- } \\
\text { tional ausgelaugt. }\end{array}$ & 0,83 & 0,85 \\
\hline Wohlbefinden & 4 & ... bin ich fröhlich. & 0,93 & 0,94 \\
\hline
\end{tabular}

Anmerkungen: $\omega_{\mathrm{R}}=$ Kongenerische Reliabilität der Referendar*innen, $\omega_{\mathrm{L}}=$ Kongenerische Reliabilität der Lehrkräfte mit zweitem Staatsexamen; 5-stufige Likert-Skala von 1=,nie“ bis 5=,,sehr oft“; Abfrage für die Lehrkräfte mit 2. Staatsexamen in Vergangenheitsform. Adaptation der Items bzgl. der physischen und psychischen Symptome sowie des Wohlbefindens nach Lohaus et al. (2018), die Items für die emotionale Erschöpfung wurden von Maslach und Jackson (1981) übernommen.

\subsection{4 Überprüfung des Messmodells}

Die psychometrischen Eigenschaften des verwendeten Erhebungsinstrumentes wurden mittels konfirmatorischer Faktorenanalysen geprüft. Zum Einsatz kam hierbei ein robuster ML-Schätzer mit Yuan-Bentler- $\chi^{2}$-Korrektur (Yuan und Bentler 2000) sowie robusten Standardfehlern und skalierten Fitindizes. Folgende Schwellenwerte zur Überprüfung der globalen Modellgüte wurden angelegt: $C F I>0,90$, $R M S E A<0,07$ und SRMR $<0,08$ (Hair et al. 2018). Als Maß für die kongenerische Reliabilität der Skalen wird der Koeffizient $\omega$ angegeben (siehe Tab. 2 und 3 und 4). Die Berechnungen wurden mit dem R-Paket lavaan 0.6-6 durchgeführt (Rosseel 2012; R Core Team 2021).

Das geschätzte Messmodell, wie es in den Abschn. 3.2.1-3.2.3 beschrieben wurde, weist eine gute bis sehr gute Passung bezüglich der Daten der Referendar*innen sowie der Lehrkräfte auf: $\chi_{R}^{2}(900)=2630,20, p_{R}<0,001, C F I_{R}=0,95$, $R M S E A_{R}=0,03\left(\mathrm{CI}_{95 \%}=0,033\right.$ bis 0,036$), S R M R_{R}=0,04$ sowie $\chi_{L}^{2}(900)=1905,95$, $p_{L}<0,001, C F I_{L}=0,94, R M S E A_{L}=0,04\left(\mathrm{CI}_{95} \%=0,035\right.$ bis 0,040$), S R M R_{L}=0,05$. Die kongenerischen Reliabilitäten weisen auf hohe interne Konsistenzen der einzelnen Skalen hin (zumeist $\omega>0,70)$. Ausschließlich die Skalen Ambivalenz $\left(\omega_{\mathrm{R}}=\omega_{\mathrm{L}}=0,64\right)$ sowie Belastung durch Kolleg*innen $\left(\omega_{\mathrm{R}}=0,62, \omega_{\mathrm{L}}=0,65\right)$ weisen etwas niedrigere Reliabilitäten auf. Aufgrund der insgesamt zufriedenstellenden psychometrischen Eigenschaften des Messmodells lässt sich dieses zur Prüfung der Untersuchungshypothesen nutzen.

\subsection{Auswertungsstrategie}

Zur Überprüfung der Hypothesen bedarf es der Testung direkter sowie moderierter Zusammenhänge zwischen latenten Variablen. Zur konfirmatorischen Schätzung messfehlerbereinigter Parameter für derartige Zusammenhänge werden in der Literatur verschiedene Vorgehensweisen beschrieben (z.B. Klein und Moosbrugger 2000; Little et al. 2006; Marsh et al. 2007). Im vorliegenden Beitrag wird ein latent moderierter Strukturgleichungsansatz (latent moderated structural equations, LMS) gewählt. Der Vorteil dieses Ansatzes liegt darin, dass sowohl für die direkten latenten Pfadkoeffizienten als auch für die latenten Interaktionsterme jeweils nur ein einziger 
Parameter geschätzt werden muss, weshalb das Verfahren als effizient eingeschätzt werden kann (Maslowsky et al. 2015). Darüber hinaus ist das Verfahren in Mplus als gängiger Analysesoftware für latente Modelle implementiert (Asparouhov und Muthén 2019), welche in der vorliegenden Analyse in der Version 8.2 verwendet wurde.

Bei der Schätzung des LMS wird den beschriebenen Vorgehensweisen in Maslowsky et al. (2015) gefolgt. In einem ersten Schritt wird ein Strukturgleichungsmodell ohne die Interaktionsterme geschätzt. Dieses Modell wird zur Überprüfung der globalen Modellpassung anhand der im Kontext der konfirmatorischen Faktorenanalyse angelegten Schwellenwerte von CFI, RMSEA und SRMR genutzt. In einem zweiten Schritt wird das gleiche Modell ergänzt durch die Interaktionsterme geschätzt. Diese Modellschätzung erlaubt die Ausgabe der final zu interpretierenden Modellparameter. Da bei den Modellen mit Interaktionstermen keine globalen Fitindizes berechnet werden können, werden zur Einschätzung der Modellgüte das Akaike (AIC) und das Bayesianische $(B I C)$ Informationskriterium angegeben. Zusätzlich wird ein LogLikelihood-Test berechnet. Ein signifikantes Ergebnis dieses Tests weist darauf hin, dass die globale Modellgüte des Interaktionsmodells ähnlich gut oder besser ist als die Güte des Modells ohne Interaktionen (Maslowsky et al. 2015).

Die drei potenziellen Belastungsquellen gehen jeweils als latente Faktoren erster Ordnung in die Modellierung ein. Die abhängige Variable Beanspruchungsreaktionen wird als latenter Faktor zweiter Ordnung reflexiv mit den latenten Faktoren erster Ordnung (a) physische Symptome, (b) emotionale Erschöpfung und (c) Wohlbefinden als Indikatoren operationalisiert. Dieses Vorgehen erlaubt eine sparsame Modellschätzung, in der nur eine einzige abhängige Variable pro Modell spezifiziert wird. Zur weiteren Reduktion der Anzahl der insgesamt zu schätzenden Modelle wird ein weiterer latenter Faktor zweiter Ordnung reflexiv mit den latenten Faktoren erster Ordnung (a) Transparenz, (b) Fairness und (c) Vertrauen als Indikatoren gebildet. Dieser Faktor operationalisiert die Gesamtheit der Beziehungsqualitäten zwischen Referendar*in und deren Seminarlehrkraft mit positiver Valenz. Er wird im weiteren Verlauf unter der Bezeichnung „Beziehungsfacetten mit positiver Valenz" geführt. Wie sich zeigen wird, ist dieses Vorgehen aufgrund der hohen Korrelationen der jeweiligen Indikatoren gerechtfertigt (siehe Abschn. 4.1). Die erlebte beziehungsbezogene Ambivalenz als Beziehungsfacette mit negativer Valenz wird als latenter Faktor erster Ordnung modelliert.

Als Kontrollvariablen gehen das Alter der Befragten sowie deren Geschlecht in die Modellschätzungen ein. Das erstgenannte Merkmal wird einbezogen, um für in der Literatur beschriebene Alterseffekte im Beanspruchungs- und Stresserleben zu kontrollieren (z. B. Stefaniak et al. 2021). Letztere Variable wird als Kontrollvariable aufgenommen, da vorliegende Studien auf geschlechterspezifische Unterschiede in der Stresswahrnehmung und -entstehung hinweisen (z. B. Chaplain 2008; Lohaus et al. 2004; Sauro et al. 2003). 


\section{Empirische Befunde}

\subsection{Deskriptive Ergebnisse und Korrelationen}

Tab. 5 stellt die deskriptiven Informationen zu den Skalen für beide Teilstichproben zur Verfügung. Die Produktvariablen aus Belastungs- und Relevanzeinschätzung bezüglich der jeweiligen Belastungsquellen zeigen, dass die Kerntätigkeiten des Vorbereitungsdienstes $\left(M_{R}=6,96, S D_{R}=2,03 ; M_{L}=6,70, S D_{L}=2,10\right) \mathrm{im}$ Vergleich zum Umgang mit den Schüler*innen $\left(M_{R}=3,91, S D_{R}=1,80 ; M_{L}=3,79, S D_{L}=1,79\right)$ und den Kolleg*innen $\left(M_{R}=3,53, S D_{R}=2,21 ; M_{L}=4,03, S D_{L}=2,43\right)$ im Mittel als am stärksten beanspruchend wahrgenommen werden bzw. wurden. Im Stichprobenmittel erleben bzw. erlebten die Befragten die Beziehung zur Seminarlehrkraft im Vorbereitungsdienst tendenziell als transparent $\left(M_{R}=4,39, S D_{R}=1,07 ; M_{L}=4,14\right.$, $\left.S D_{L}=1,23\right)$, fair $\left(M_{R}=4,80, S D_{R}=1,06 ; M_{L}=4,53, S D_{L}=1,27\right)$ und vertrauensvoll $\left(M_{R}=3,96, S D_{R}=1,20 ; M_{L}=3,73, S D_{L}=1,30\right)$. Ähnlich hoch beurteilten die Befragten jedoch auch die erlebte Ambivalenz der Beziehung $\left(M_{R}=3,73, S D_{R}=1,05\right.$; $\left.M_{L}=3,70, S D_{L}=1,09\right)$. Physische Symptome werden bzw. wurden von den befragten (ehemaligen) Referendar*innen seltener erlebt $\left(M_{R}=2,51, S D_{R}=0,84 ; M_{L}=2,45\right.$, $\left.S D_{L}=0,86\right)$ als emotionale Erschöpfungssymptome $\left(M_{R}=3,73, S D_{R}=0,87 ; M_{L}=3,76\right.$, $\left.S D_{L}=0,91\right)$. Gleichzeitig berichten die Befragten ein gewisses Maß an Wohlbefinden $\left(M_{R}=3,36, S D_{R}=0,82 ; M_{L}=3,25, S D_{L}=0,84\right)$.

Bei den Skalenkorrelationen zeigen sich hinsichtlich der jeweiligen Effektstärken mehrheitlich moderate positive Zusammenhänge zwischen den drei Belastungsquellen $\left(r_{R}=0,24\right.$ bis 0,$43 ; r_{L}=0,05$ bis 0,33$)$, welche ihrerseits bei den Referendar*innen durchweg und bei den Lehrkräften mehrheitlich signifikant positiv mit den physischen Symptomen $\left(r_{R}=0,10\right.$ bis 0,$56 ; r_{L}=0,04$ bis 0,55$)$ und der emotionalen Erschöpfung $\left(r_{R}=0,25\right.$ bis 0,$82 ; r_{L}=0,18$ bis 0,84$)$ sowie mehrheitlich signifikant negativ mit dem im Referendariat erlebten Wohlbefinden $\left(r_{R}=-0,11\right.$ bis $-0,58 ; r_{L}=-0,08$ bis $-0,59)$ korrelieren. Die betragsmäßig stärksten Zusammenhänge weisen die drei genannten Beanspruchungsreaktionen mit der Belastung bedingt durch die Kerntätigkeit auf.

Die Beziehungsfacetten Transparenz, Fairness und Vertrauen korrelieren jeweils signifikant negativ mit der Belastung durch die Kerntätigkeit $\left(r_{R}=-0,24\right.$ bis $-0,25$; $r_{L}=-0,11$ bis $\left.-0,17\right)$ sowie durch Kolleg*innen $\left(r_{R}=-0,15\right.$ bis $-0,23 ; r_{L}=-0,25$ bis $-0,30)$. Die Beziehungsfacetten korrelieren positiv mit der Belastung durch Schüler*innen $\left(r_{R}=0,05\right.$ bis 0,$08 ; r_{L}=0,13$ bis 0,16$)$, wobei sich jedoch nicht alle Korrelationen signifikant von Null unterscheiden. Bezüglich der erlebten Ambivalenz zeigen sich jeweils umgekehrte Zusammenhänge: Sie korreliert signifikant positiv mit der Belastung durch die Kerntätigkeit $\left(r_{R}=0,38 ; r_{L}=0,40\right)$ sowie durch Kolleg*innen $\left(r_{R}=0,21 ; r_{L}=0,26\right)$. Zwischen Ambivalenz und Belastung durch Schüler*innen besteht kein signifikanter Zusammenhang $\left(r_{R}=-0,01 ; r_{L}=-0,03\right)$. Die Beziehungsfacetten Transparenz, Fairness und Vertrauen korrelieren stark und positiv miteinander $\left(r_{R}=0,89\right.$ bis 0,$94 ; r_{L}=0,92$ bis 0,95$)$ und stehen mit der erlebten Ambivalenz in negativem Zusammenhang $\left(r_{R}=-0,58\right.$ bis $-0,68 ; r_{L}=-0,53$ bis $-0,65)$. Hinsichtlich der Beanspruchungsreaktionen zeigen sich signifikant negative Zusammenhänge zwischen Transparenz, Fairness sowie Vertrauen und den physi- 


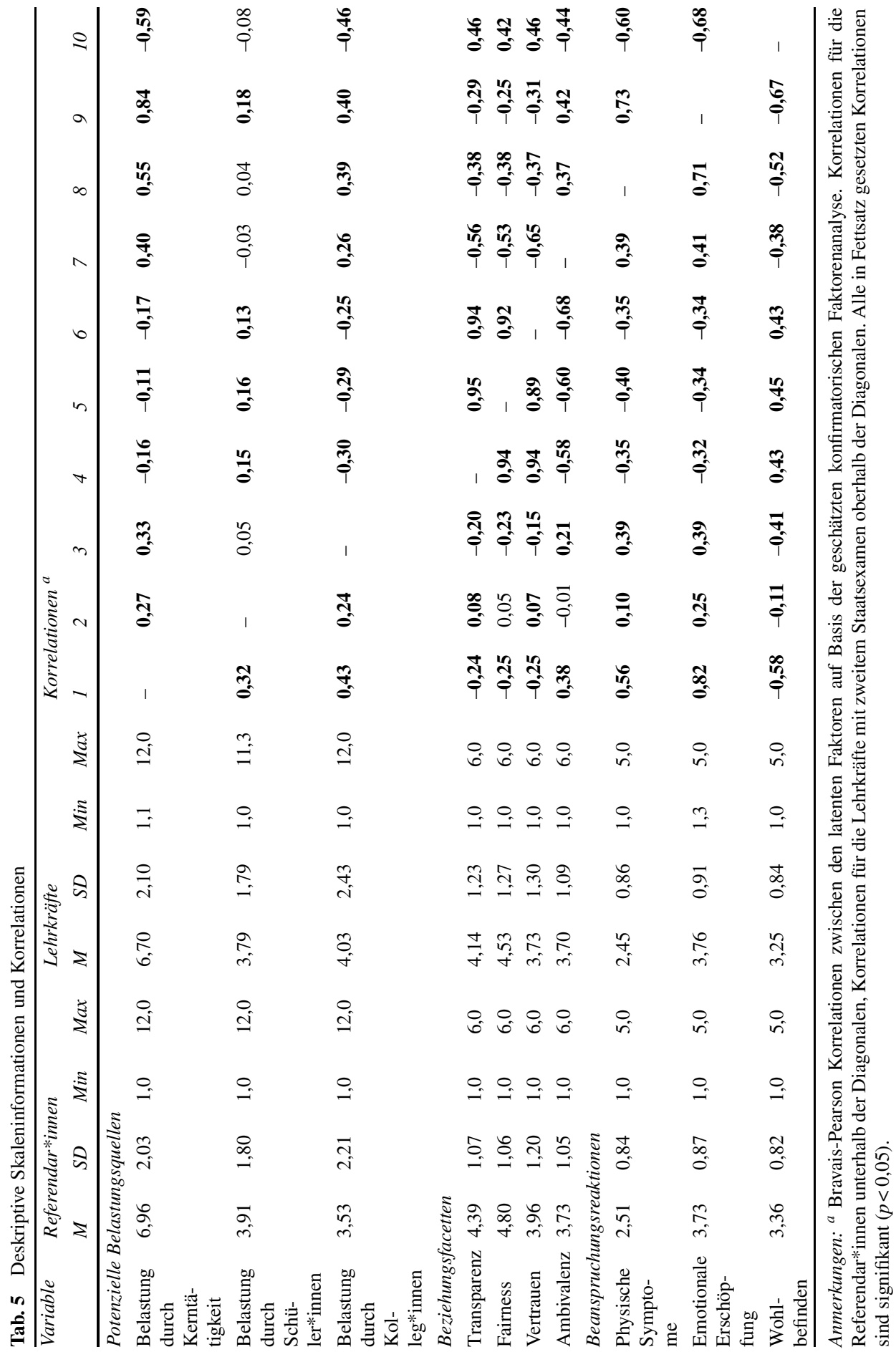


schen Symptomen $\left(r_{R}=-0,35\right.$ bis $-0,40 ; r_{L}=-0,37$ bis $\left.-0,38\right)$ sowie der emotionalen Erschöpfung $\left(r_{R}=-0,32\right.$ bis $-0,34 ; r_{L}=-0,25$ bis $\left.-0,31\right)$ und signifikant positive Zusammenhänge mit dem Wohlbefinden $\left(r_{R}=0,43\right.$ bis 0,$45 ; r_{L}=0,42$ bis 0,46$)$. Die im Rahmen der Beziehung erlebte Ambivalenz steht mit den beiden erst genannten Beanspruchungsreaktionen in signifikant positivem Zusammenhang ( $r_{R}=0,39$ und 0,$41 ; r_{L}=0,37$ und 0,42 ) und korreliert signifikant negativ mit dem Wohlbefinden $\left(r_{R}=-0,38 ; r_{L}=-0,44\right)$.

\subsection{Ergebnisse der latenten Moderationsanalysen}

Die Ergebnisse der LMS-Analysen sind in Tab. 6 dargestellt. Im ersten Schritt wurde jeweils ein Modell zur Erklärung der Beanspruchungsreaktionen im Referendariat unter ausschließlichem Einbezug der Kontrollvariablen Geschlecht und Alter für die Teilstichprobe der Referendar*innen $\left(\mathrm{M}_{R} 0\right)$ sowie der Lehrkräfte $\left(\mathrm{M}_{\mathrm{L}} 0\right)$ gerechnet. Bei den Referendar*innen und den Lehrkräften zeigen sich sowohl bei den Variablen Geschlecht $\left(\beta_{\mathrm{MR} 0}=0,05, p=0,034 ; \beta_{\mathrm{ML} 0}=0,12, p=0,001\right)$ also auch Alter $\left(\beta_{\mathrm{MR} 0}=0,09, p<0,001 ; \beta_{\mathrm{ML} 0}=0,07, p=0,043\right)$ signifikant positive Effekte, die jedoch als klein einzuschätzen sind: Frauen erleben im Durchschnitt etwas mehr Beanspruchung im Vergleich zu Männern und je älter die Befragten sind, desto eher wird das Referendariat mit Beanspruchungsreaktionen in Verbindung gebracht. Das Modell erklärt jedoch in beiden Teilstichproben fast keine Varianz $\left(R^{2}{ }_{M R O}=0,010 ; R^{2}{ }_{M L O}=0,017\right)$ und weist einen vergleichsweise schlechten ModellFit auf $\left(R M S E A_{M R O}=0,121 ; R M S E A_{M L O}=0,092\right)$.

In einem zweiten Schritt wurden mit den Daten der Referendar*innen sowie der Lehrkräfte separat für die Beziehungsfacetten mit positiver Valenz (Faktor zweiter Ordnung: Transparenz, Vertrauen, Fairness) sowie die Beziehungsfacette mit negativer Valenz (Ambivalenz) jeweils ein Modell ohne Interaktionsterme $\left(M_{R} 1 \mathrm{a}\right.$ und $M_{R}$ 2a sowie $M_{L} 1 a$ und $\left.M_{L} 2 a\right)$ sowie ein Modell mit Interaktionstermen $\left(M_{R} 1 b\right.$ und $M_{R} 2 b$ sowie $M_{L} 1 b$ und $M_{L} 2 b$ ) geschätzt (siehe auch Abb. 1). Die ausgegebenen Fitindizes weisen bei den Modellen ohne Interaktionsterme jeweils auf einen akzeptablen bis guten Modellfit hin $(C F I=0,925$ bis 0,939, $R M S E A=0,037$ bis 0,041, $S R M R=0,046$ bis 0,058). Evidenz für eine ähnliche gute Passung der Modelle mit Interaktionstermen finden sich für die Beziehungsfacetten mit positiver und negativer Valenz aufgrund der signifikanten Log-Likelihood-Tests (jeweils $p<0,001$ ) sowie den im Vergleich jeweils geringeren $A I C$ - und $B I C$-Werten. Die Varianzaufklärung fällt bei allen Modellen hoch aus $\left(R^{2}=0,727\right.$ bis 0,826).

Die Pfadkoeffizienten der Kontrollvariablen in beiden Teilstichproben sind größtenteils vergleichbar mit denen der jeweiligen Basismodelle $\mathrm{M}_{R} 0$ und $\mathrm{M}_{\mathrm{L}} 0$ : Das Geschlecht hat jeweils einen signifikant positiven Einfluss auf die Beanspruchungsreaktionen $\left(\beta_{\mathrm{R}}=0,06\right.$ bis 0,07 und $\beta_{\mathrm{L}}=0,09$ bis $\left.0,10, p<0,01\right)$. Die Effekte des Alters fallen hingegen ausschließlich bei den Referendar*innen bei der Beziehungsfacette Ambivalenz signifikant aus. In den entsprechenden Modellen $M_{R} 2 a$ und $M_{R} 2 b$ zeigt sich ein signifikant positiver Effekt des Alters $\left(\beta_{\mathrm{MR} 2 \mathrm{a}}=0,06, p=0,006 ; \beta_{\mathrm{MR} 2 \mathrm{~b}}=0,03\right.$, $p=0,005)$.

Die Haupteffekte der Belastungsquellen und der Beziehungsfacetten zeigen in allen geschätzten Modellen in beiden Teilstichproben ein ähnliches Bild: 


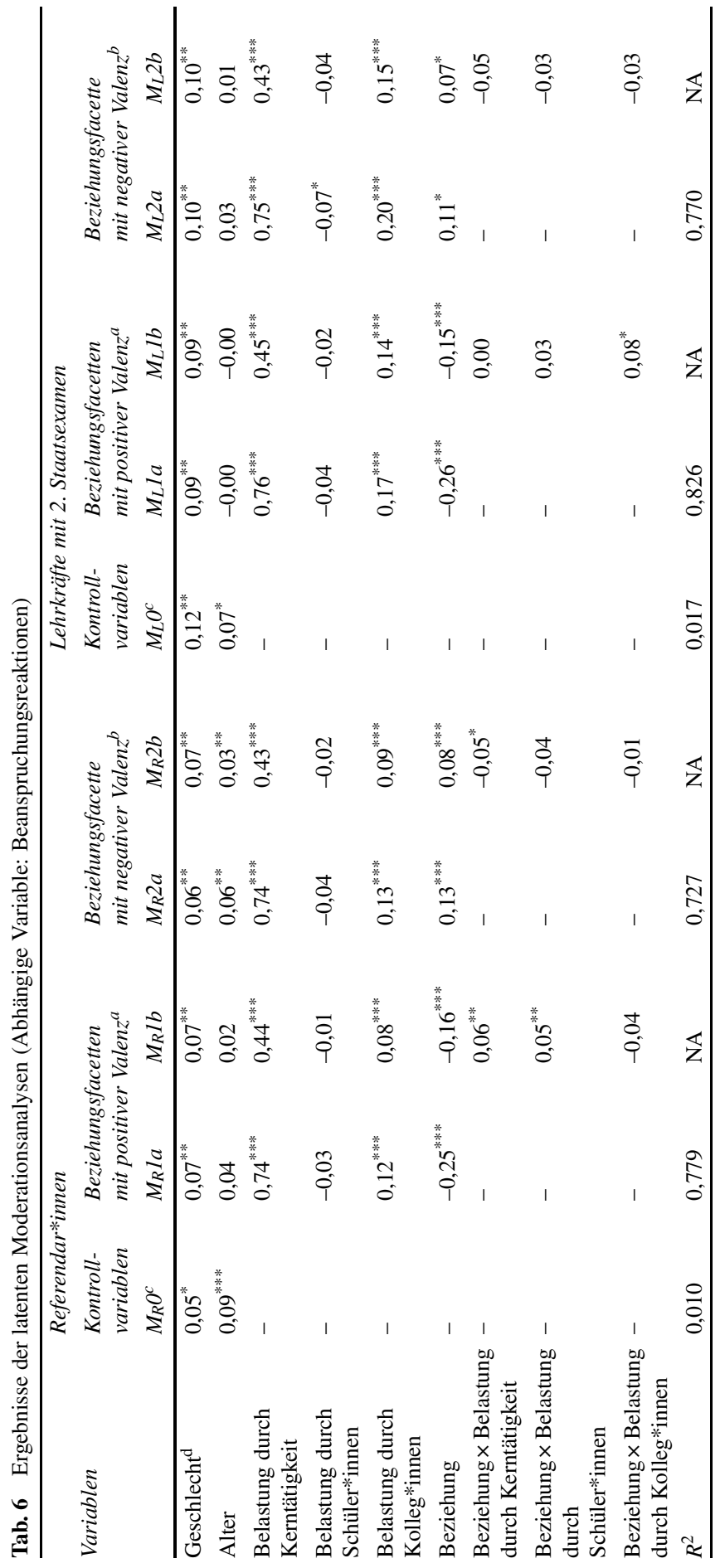




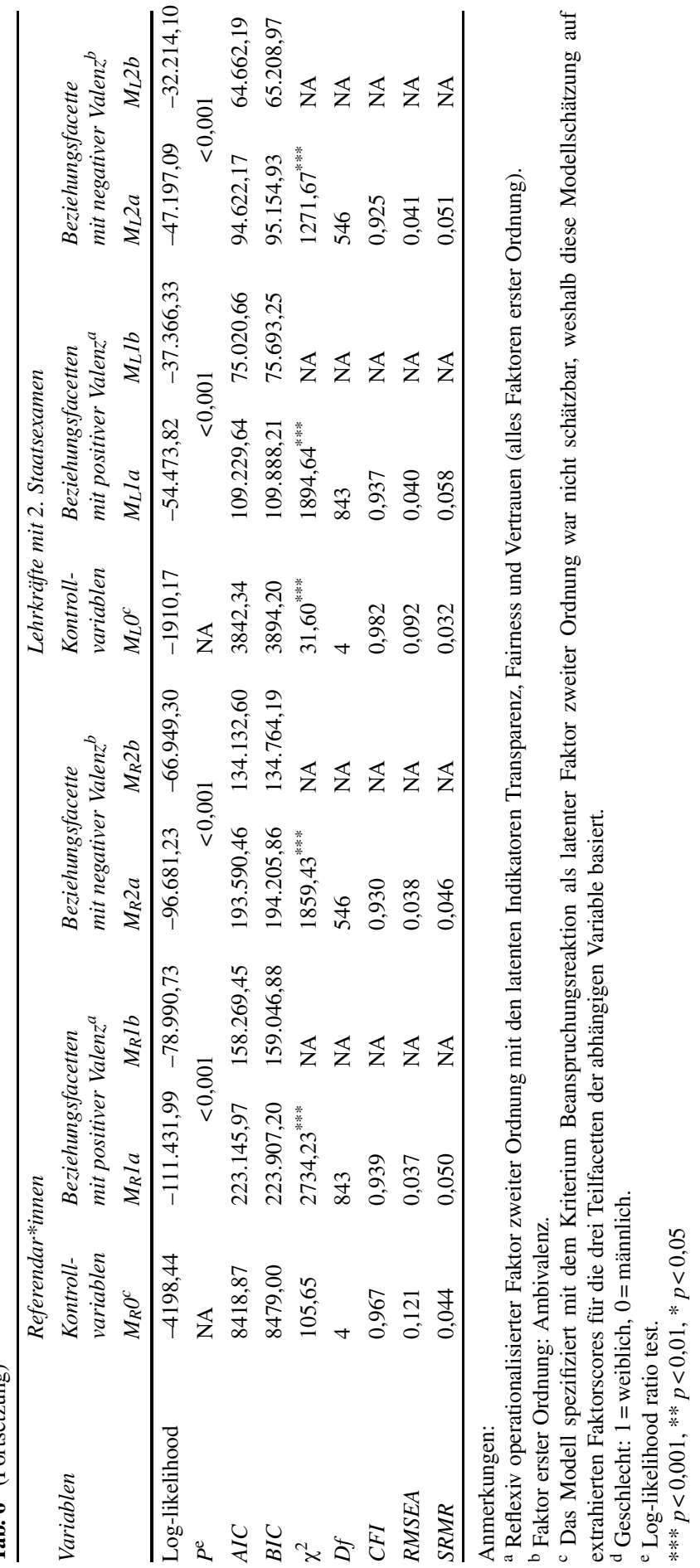


a. Die Belastung aufgrund der Kerntätigkeit sagt Beanspruchungssreaktionen im Vorbereitungsdienst signifikant und stark voraus $\left(\beta_{R}=0,43\right.$ bis 0,74 und $\beta_{L}=0,43$ bis $0,76, p<0,001)$.

b. Die Belastung aufgrund des Umgangs mit Schüler*innen wirkt sich kaum auf die Beanspruchungsreaktionen aus. Nur einer der geschätzten Pfadkoeffizienten unterscheidet sich signifikant von Null $\left(\beta_{\mathrm{ML} 2 \mathrm{a}}=-0,07, p=0,049\right)$.

c. Das Belastungserleben, ausgelöst durch die Kolleg*innen im Lehrer*innenkollegium, wirkt sich in allen Modellen signifikant positiv auf die wahrgenommenen Beanspruchungssreaktionen im Vorbereitungsdienst aus $\left(\beta_{R}=0,08\right.$ bis 0,13 und $\beta_{\mathrm{L}}=0,14$ bis $\left.0,20, p<0,001\right)$.

d. Für die Beziehungsqualität mit positiver Valenz (gemeinsamer Faktor zweiter Ordnung aus Transparenz, Fairness und Vertrauen) zeigen sich jeweils signifikant negative Einflüsse auf die Beanspruchungsreaktionen im Vorbereitungsdienst $\left(\beta_{\mathrm{R}}=-0,16\right.$ bis $-0,25$ und $\beta_{\mathrm{L}}=-0,15$ bis $\left.-0,26, p<0,001\right)$.

e. Für die Beziehungsfacette Ambivalenz wurden hingegen signifikant positive Effekte auf die Symptomatik geschätzt $\left(\beta_{R}=0,08\right.$ bis 0,13 und $\beta_{L}=0,07$ bis 0,11 , $p<0,05)$.

f. In den Modellen mit Interaktionseffekten weisen die geschätzten Pfadkoeffizienten durchgehend kleinere Werte als in den Modellen ohne Interaktionseffekte auf, bleiben jedoch weitgehend signifikant und hinsichtlich der jeweiligen Effektrichtung erhalten.

Im Vergleich zu den übrigen signifikanten Pfadkoeffizienten der geschätzten Modelle zeigen sich nur geringe Einflüsse der Interaktionsterme. Durchweg signifikant, jedoch bei sehr geringen Effektstärken, wird nur der Zusammenhang zwischen der Belastung aufgrund der Kerntätigkeit und der Beanspruchungsreaktionen in der Stichprobe der Referendar*innen durch die einzelnen Beziehungsfacetten moderiert $\left(\beta_{\mathrm{MR} 1 \mathrm{~b}}=0,06, p=0,005 ; \beta_{\mathrm{MR} 2 \mathrm{~b}}=-0,05, p=0,020\right)$. Je transparenter, fairer, vertrauensvoller und weniger ambivalent die Beziehung zu den Seminarlehrkräften erlebt wird, umso stärker wirkt sich demnach die Belastung, ausgelöst durch die Kerntätigkeit des Vorbereitungsdienstes, auf die Manifestation von Beanspruchungsreaktionen aus. Signifikante Moderationseffekte zeigen sich ansonsten noch zwischen der Belastung durch die Schüler*innen bei den Referendar*innen $\left(\beta_{\mathrm{MR} 1 \mathrm{~b}}=0,05, p=0,003\right)$ sowie der Belastung durch die Kolleg*innen bei den Lehrkräften und der Beanspruchungsreaktionen für die positiv konnotierten Beziehungsfacetten $\left(\beta_{\mathrm{ML} 1 \mathrm{~b}}=0,08\right.$, $p=0,021)$.

\section{Diskussion}

\subsection{Zusammenfassung der Befunde und Rückbezug zu den Hypothesen}

Entsprechend der ersten Hypothese wurden signifikant positive Haupteffekte der im Referendariat erlebten Belastung durch die Kerntätigkeit (starke Effekte) sowie durch den Umgang mit Kolleg*innen im Lehrer*innenkollegium (schwache Effekte) auf die Beanspruchung identifiziert. Entgegen der Annahme, dass sich auch Belas- 
tungsfaktoren, welche sich auf den Umgang mit Schüler*innen beziehen, positiv auf die Beanspruchung auswirken, zeigen sich diesbezüglich in der Stichprobe vernachlässigbare, zum Teil nicht signifikante, negative Effekte. Die Ergebnisse weisen daher darauf hin, dass die Beanspruchung im Referendariat im Wesentlichen durch die Kerntätigkeit angehender Lehrkräfte (z. B. Unterricht vorbereiten und selbstständig durchführen) erklärt werden kann (ähnlich Horstmeyer 2018). Zurückzuführen mag dies auf Phänomene sein, die in der Literatur unter dem Begriff des sogenannten Praxisschocks diskutiert und erforscht werden (z. B. Dicke et al. 2016). Im Vergleich zu erfahrenen Lehrpersonen (Experten) erfordert beispielsweise die Unterrichtsvorbereitung seitens der Referendar*innen (Novizen) aufgrund noch unzureichend ausgebildeter Routinen viel Zeit und Aufwand. Darüber hinaus fehlt ihnen häufig noch relevantes Erfahrungswissen, auf das in belastenden Situationen zurückgegriffen werden kann. Dadurch stehen kognitiv keine oder nur wenige Ressourcen zur adäquaten Situationsbewältigung zur Verfügung (Anspal et al. 2019; Thiel et al. 2012; Wolff et al. 2016). Verstärkt mag dies zusätzlich dadurch werden, dass in schulischen Situationen häufig spontan und unmittelbar reagiert werden muss (Doyle 2006) und auf umweltbezogene Ressourcen (z.B. Rückmeldungen im Kreise erfahrener Kolleg*innen) (noch) nicht oder nur begrenzt zurückgegriffen werden kann. Somit stehen für die potenziell als Bedrohung bewerteten Stimuli entsprechende Bewältigungsressourcen nur eingeschränkt zur Verfügung, was nach den Grundannahmen des transaktionalen Stressmodells zu entsprechenden Beanspruchungsreaktionen führt (Lazarus 1966). Verschärft wird die Situation für die Referendar*innen zusätzlich dadurch, dass sie ihre eigene Lehrtätigkeit nicht nur unter für sie neuen Bedingungen ausüben, sondern dass diese darüber hinaus bewertet und damit karrierewirksam wird. Dass Belastungen durch Kolleg*innen im Vergleich zur Kerntätigkeit weniger stark als beanspruchend erlebt werden, deckt sich mit den Befunden von Horstmeyer (2018), wonach die Belastung durch Leistungsdruck und Arbeitsbelastung höher ausfällt als die Belastung durch soziale Interaktionen. Dies mag mit der generell relativ hohen Autonomie von Lehrkräften zusammenhängen. Auch im Referendariat agieren angehende Lehrkräfte größtenteils eigenständig. Dies ermöglicht den Referendar*innen soziale Kontakte selektiv auszuwählen und beispielsweise nur zu solchen Kolleg*innen Kontakt zu suchen, die als zugänglich und unterstützend wahrgenommen werden. Die zum Teil gefundenen negativen Effekte der Belastung durch Schüler*innen können aufgrund ihrer geringen Stärke $\left(\beta_{R}=-0,01\right.$ bis $-0,04$ und $\beta_{L}=-0,02$ bis $\left.-0,07\right)$ vernachlässigt werden. Dass einer dieser Pfadkoeffizienten im Modell $\mathrm{M}_{\mathrm{L}} 2 \mathrm{~b}$ das übliche Signifikanzniveau erreicht, ist vor allem auf die große Stichprobe zurückzuführen. Dennoch überrascht, dass der Umgang mit Schüler*innen so gut wie keine Auswirkung auf die Beanspruchung im Referendariat hat. Denkbar wäre, dass die Referendar*innen ihre Aufmerksamkeit vor allem auf ihre schulische Kerntätigkeit richten und eventuell Schwierigkeiten im Umgang mit den Schüler*innen auf die eigenen Defizite in der Unterrichtsvorbereitung und -durchführung zurückführen. In dem Fall würde die Belastung aufgrund der Unterrichtsvorbereitung und -durchführung die Belastung aufgrund von Aktionen von Schüler*innen überlagern.

Im Einklang mit der zweiten Hypothese zeigen sich signifikant negative Effekte der Beziehungsfacetten Transparenz, Fairness und Vertrauen sowie ein signifikant 
positiver Effekt der Ambivalenz auf die Beanspruchung. Es bestätigt sich die Annahme, dass sich eine positiv erlebte Beziehung zu den Seminarlehrkräften im Referendariat im Sinne einer Ressource auf die Beanspruchung der Referendar*innen mindernd auswirkt (siehe Abschn. 2.2.2).

Entgegen der dritten Hypothese verdeutlichen die signifikanten Interaktionseffekte, dass je transparenter, fairer, vertrauensvoller und weniger ambivalent die Beziehung zu den Seminarlehrkräften erlebt wird, sich die durch unterschiedliche Belastungsquellen ausgelöste Belastung umso stärker auf die Beanspruchung auswirkt. Signifikante Moderationseffekte bestehen (a) zwischen der Belastung aufgrund der Kerntätigkeit des Referendariats und der Beanspruchung für die Beziehungsfacetten in der Stichprobe der Referendar*innen, (b) zwischen der Belastung durch Schüler*innen und der Beanspruchung für die Beziehungsfacetten mit positiver Valenz in der Stichprobe der Referendar*innen sowie (c) zwischen der Belastung durch Kolleg*innen und der Beanspruchung für die Beziehungsfacetten mit positiver Valenz in der Stichprobe der Lehrkräfte. Es ist anzumerken, dass die folgenden möglichen Argumente zur Erklärung der gefundenen kontraintuitiven Moderatoreffekte zunächst spekulativ bleiben müssen. Zum einen sind die betreffenden standardisierten Regressionskoeffizienten in den Größenordnungen von $-0,05$ bis 0,08 sehr klein und daher hinsichtlich der praktischen Bedeutsamkeit quasi zu vernachlässigen, zum anderen fallen die Effekte in erster Linie aufgrund der großen Stichprobe signifikant aus. Dennoch erscheint der verstärkende Effekt einer positiv erlebten Beziehung zu den Seminarlehrkräften auf die Beanspruchung im Referendariat im Sinne einer Belastung im ersten Moment paradox. Denkbar wäre, dass ein gutes Verhältnis zu den Seminarlehrkräften dazu führt, dass die Referendar*innen die geäußerte Kritik von diesen eher annehmen und somit eigene Kompetenzdefizite auch eher erkennen. Die ansonsten als irrelevant bewerteten Stimuli des Schulalltages werden dadurch relevanter und somit tendenziell herausfordernder oder sogar bedrohlicher, da negatives Feedback, welches ernst genommen und internal attribuiert wird, die Sorge schürt, ob das Referendariat erfolgreich beendet werden kann. Im Rahmen von negativ erlebten Beziehungen ist es hingegen denkbar, dass die Kritik der Seminarlehrkraft nicht an- bzw. ernst genommen und auf die negative Beziehungsqualität anstatt auf das eigene Können und Handeln attribuiert wird. In diesem Zusammenhang wäre in nachfolgenden Studien auch die Qualität des Feedbacks seitens der Seminarlehrkräfte und damit dessen Lernwirksamkeit sowie daraus resultierend eine mögliche bessere Bewältigung entsprechender Anforderungen zu untersuchen. Dies erscheint insofern relevant, da die Feedbackqualität einen weiteren Faktor des Beziehungsgefüges zwischen Referendar*in und Seminarlehrkraft darstellt, welcher in der vorliegenden Studie nicht berücksichtigt wurde.

Wie dargestellt wurden alle Analysen separat mit den Daten der Referendar*innen $\left(n_{R}=1756\right)$ wie auch der Lehrkräfte mit zweitem Staatsexamen $\left(n_{L}=826\right)$ berechnet. Die annähernde Kongruenz der Modellschätzungen in den beiden unabhängigen Teilstichproben weist auf eine hohe Robustheit der vorliegenden Befunde hin. Darüber hinaus gibt es auf Basis der Modellschätzungen keine Hinweise darauf, dass Lehrkräfte, welche das Referendariat bereits einige Zeit abgeschlossen haben, aufgrund von Erinnerungseffekten systematisch anders antworteten als Personen, die mitten im Referendariat sind. 


\subsection{Limitationen der Vorgehensweise und Implikationen für die weitere Forschung}

Wir adressieren in unserem Beitrag ein für die Lehrer*innenbildung relevantes Thema, welches in der vorliegenden Form insbesondere hinsichtlich der Operationalisierung der verwendeten belastungsbezogenen (Belastungseinschätzung und Relevanzeinschätzung) und beziehungsbezogenen Konstrukte unseres Wissens bisher nicht untersucht wurde. Dennoch weist die Studie zu benennende Limitationen auf. Diese beziehen sich erstens auf die zugrundeliegende Datenlage. Wenngleich auf eine relativ umfangreiche Stichprobe zurückgegriffen werden kann, ist diese aufgrund des verwendeten Verfahrens (Akquise über Foren und soziale Netzwerke, Schneeballsystem) nicht repräsentativ für die unterschiedlichen Bundesländer und Schularten. Aufgrund der bezüglich Bundesland und Schulart nicht repräsentativen Stichprobe verzichten wir auf entsprechende Teilanalysen, da uns dies hinsichtlich möglicher Schlussfolgerungen als nicht angemessen erscheint. Folglich wären für weiterführende Studien entsprechende bundesland- sowie schulartbezogene repräsentative Erhebungen erforderlich.

Eine weitere Limitation besteht in der Abfrage der Beziehungsitems. Da es bundeslandspezifische Besonderheiten in der Benennung der Seminarlehrkräfte gibt sowie die betreffenden Personen in den Bundesländern teils unterschiedliche Funktionen und Tätigkeitsbereiche haben, sind hierbei mögliche Verzerrungen bezogen auf das Erhebungsformat zu bedenken. Zwar wurde versucht, diese Problematik durch einen entsprechenden standardisierten Einführungstext im Fragebogen aufzufangen, jedoch sollten die diesbezüglichen Einschätzungen in einer zukünftigen Studie differenzierter erfolgen.

Zum Dritten wurden soziodemographische Merkmale nur sparsam abgefragt, um einerseits den Fragebogen nicht zu verlängern und andererseits die Anonymität der Teilnehmer*innen zu wahren. Es bleibt daher offen, inwiefern spezielle private Umstände (z.B. zu versorgende Kinder oder zu pflegende Verwandte) oder auch andere persönliche Merkmale (bspw. Leistung im Studium, sozioökonomischer Hintergrund oder relevante Persönlichkeitsmerkmale bzw. Dispositionen) zusätzlichen Einfluss auf die Beanspruchungsreaktionen sowie die untersuchten Zusammenhänge haben. Dem müsste ebenfalls in Folgestudien nachgegangen werden.

Eine vierte Limitation bezieht sich auf das querschnittliche Design der Datenerhebung. Dieser Ansatz ermöglicht es zwar, mittels strukturprüfender Verfahren Zusammenhänge zwischen unterschiedlichen Variablen zu identifizieren, Aussagen über (zeitlich) kausale Interdependenzen sind damit jedoch nicht möglich. Einerseits deswegen nicht, da hierfür längsschnittliche Daten erforderlich wären, um beispielsweise zeitliche Dynamiken in der Genese der professionellen Beziehung zwischen Referendar*innen und deren Seminarlehrkräften über den Ausbildungsverlauf hinweg abzubilden. Andererseits deswegen nicht, da lediglich die Selbstauskunft der Referendar*innen als Datenquelle verwendet wird und keine endogenen Variablen, wie beispielsweise Strukturinformationen zu den genauen institutionellen Ausbildungsbedingungen, keine leistungsbezogenen Daten (z. B. Teil- oder Abschlussnoten) und keine Daten zur Fremdeinschätzung der betrachteten Beziehung seitens der Seminarlehrkräfte (mögliche Abweichungen zur Einschätzung der Refe- 
rendar*innen; Leshem 2012; Manning und Hobson 2017) erhoben wurden. Längsschnittliche Erhebungs- und insbesondere videogestützte Beobachtungsverfahren sowie zeitreihenanalytische Methoden sind in diesem Zusammenhang perspektivisch als geeignet anzusehen, um die in konkreten pädagogischen Handlungen und deren Reflexion auftretenden Oszillationen innerhalb der antinomischen Spannungsfelder näher zu untersuchen.

\subsection{Schlussfolgerung für die Ausbildungspraxis: Beziehungsarbeit im Referendariat als zentraler Ausbildungsbestandteil}

Entsprechend der Relevanz der professionellen pädagogischen Beziehung zwischen Referendar*in und Seminarlehrkraft für die in der zweiten Phase der Lehrer*innenausbildung resultierende Beanspruchung wäre der betreffenden Beziehung in der Ausbildungspraxis ein stärkeres Gewicht beizumessen. Fachliche sowie persönliche Unterstützungsleistungen der Seminarlehrkräfte sind hierbei im Spannungsverhältnis zwischen Nähe versus Distanz, Machtausübung versus Partizipation sowie Heteronomie versus Autonomie genau auszutarieren und an die individuellen Bedürfnisse der Referendar*innen sowie an die jeweilige Situation anzupassen (Harder 2014; Helsper 2004; Hennissen et al. 2008; Oser 1996; Sembill 1995).

Insofern sollten Seminarlehrkräfte auf ihre beziehungsbezogenen Aufgaben vorbereitet werden, um ihre Rolle als Mentor*innen zu finden und entsprechend professionell und reflektiert handeln zu können (Cain 2009; Kupila et al. 2017; Leshem 2012; Manning und Hobson 2017), beispielsweise durch Weiterbildungen, prozessbegleitende Supervision oder kollegiale Fallberatung. Dabei sollten die Aspekte Transparenz (z. B. in Bezug auf offen kommunizierte und partizipativ definierte Bewertungskriterien sowie konstruktive Kritik), Fairness (z. B. durch Anerkennung von Leistung und einen respektvollen Umgang) sowie Vertrauen (z. B. durch einen wertschätzenden und empathischen Umgang) berücksichtigt und vor dem Hintergrund eines potenziell ambivalenten Beziehungsgefüges reflektiert werden.

Eine Sichtung von Fortbildungsangeboten für Seminarlehrkräfte förderte lediglich für sieben Bundesländer entsprechende Fortbildungen zutage, welche sich im weitesten Sinne auf die Beziehung zwischen Seminarlehrkraft und Referendar*in beziehen. ${ }^{4}$ Von den insgesamt 22 gefundenen Fortbildungen stellt Bayern die meisten Angebote zur Verfügung. Die häufigsten Themen hinsichtlich der Vorbereitung auf die Begleitung und Betreuung von Referendar*innen stellen die Beratung von Referendar*innen, die Unterrichtsbeobachtung sowie die Unterrichtsgestaltung dar. Allerdings steht die Beratung von Referendar*innen häufig im Zusammenhang mit den fachlichen Aspekten der Beziehung zwischen Referendar*in und Seminarlehrkraft und fällt somit eher fach- bzw. unterrichts- und weniger genuin beziehungsbezogen aus. Die Gestaltung der Zusammenarbeit und der persönlichen Beziehung

\footnotetext{
4 Bei der internetbasierten Suche im Fortbildungsangebot für Lehrkräfte wurden in allen Bundesländern die Begriffe „Ausbildungslehrer*in“, „Seminarlehrer*in“, „Ausbildungslehrperson“, „Seminarlehrperson“, „Mentor*in“ und „Mentorentätigkeit“ jeweils in Verbindung mit dem Begriff „Referendariat“ verwendet. Anschließend wurden die gefundenen Fortbildungen gesichtet und nach ihren jeweiligen inhaltlichen Schwerpunkten gruppiert.
} 
zwischen Seminarlehrkraft und Referendar*in ist hingegen lediglich Gegenstand von zwei gefundenen Fortbildungen. So bietet etwa Bayern eine Gruppensupervision für Seminarlehrkräfte an, welche u. a. Themen wie die richtige Balance zwischen Kontrolle und Freiraum, das Geben von adäquatem Feedback und den Umgang mit „schwierigen“ Referendar*innen beinhaltet (FIBS 2020).

Neben dem Fokus auf der professionellen pädagogischen Beziehungsgestaltung wäre in weiterführenden Überlegungen auch die generelle Struktur des Referendariats und hier insbesondere die institutionalisierten Funktionen von Seminarlehrkräften in den Blick zu nehmen. So könnte beispielsweise über eine personelle Entkoppelung von Beratungs- und Beurteilungsfunktionen nachgedacht werden, was jedoch vor dem Hintergrund der Machbarkeit zu erörtern wäre. Entsprechende Nachteile einer nicht vorhandenen Rollentrennung wurden im vorliegenden Beitrag angesichts der benannten Doppelrolle der Seminarlehrkräfte und der damit einhergehenden Rollenambiguität und seitens der Referendar*innen erlebten Ambivalenz beschrieben. Jedoch geht mit der Doppelfunktion auch der Vorteil einher, dass Seminarlehrkräfte aufgrund ihrer Einbindung in die Beurteilungen die Referendar*innen gezielter auf entsprechende Prüfungen vorbereiten können. Weiterhin stehen die Referendar*innen in entsprechenden Beurteilungs- bzw. Prüfungssituationen nicht nur unbekannten Prüfenden gegenüber, sondern auch den ihnen bekannten Seminarlehrkräften, was - eine positive Beziehung zu diesen vorausgesetzt - Sicherheit geben kann.

Angesichts der Bedeutung der Seminarlehrkräfte für die Ausbildung von Referendar*innen in der zweiten Phase der Lehrer*innenausbildung und in Anbetracht der in diesem Beitrag dargestellten Herausforderungen, die mit dieser Aufgabe einhergehen, sollten entsprechende Angebote zur Fort- und Weiterbildung flächendeckend eingeführt, ausgebaut und institutionalisiert werden. Grundlage hierfür könnten insbesondere weiterführende empirische Studien zu Faktoren sein, welche in spezifischen Fällen zu einer besonders guten bzw. schlechten Beziehungsqualität beitragen. Dies bietet wiederum mögliche Ansatzpunkte für Interventionen zur „Beziehungsarbeit“" zwischen Referendar*innen und ihren Seminarlehrkräften. Dies erscheint in einer weiter gefassten kritisch-emanzipatorischen Perspektive insbesondere vor dem Hintergrund institutionalisierter Machtasymmetrien zwischen den betrachteten Beziehungspartner*innen und daraus entstehenden Abhängigkeitsverhältnissen relevant. Entsprechende Asymmetrien spiegeln sich hierbei im Spannungsfeld von Machterhalt und der Suche nach Autonomie sowie in entsprechenden Neben- und Folgeeffekten für persönliche Entwicklungsmöglichkeiten und -verläufe der Referendar*innen wider. Ein erster Schritt könnte demnach in der Enttabuisierung von bestehenden Macht- und Abhängigkeitsverhältnissen in der zweiten Phase der Lehrer*innenbildung sowie in der transparenten und reflektierenden Auseinandersetzung mit diesen liegen (vgl. Sembill und Kärner 2020). Denn auch Referendar*innen werden irgendwann selbst zu Lehrpersonen, welche pädagogische Beziehungen zu ihren Schüler*innen gestalten und damit Einfluss auf deren Erleben, Befinden, Handeln und Entwicklung nehmen.

Danksagung Die Autor*innen danken den anonymen Gutachter*innen sowie den beteiligten Herausgeber*innen herzlich für ihre konstruktiven Rückmeldungen, welche zur Optimierung des Artikels beigetragen haben. Weiterhin danken die Autor*innen den Befragungsteilnehmer*innen, welche durch ihr 
Engagement einen wichtigen Beitrag zur Forschung zur zweiten Phase der Lehrer*innenbildung geleistet haben.

Förderung Das Projekt wurde vom „Young Scholar Fund im Rahmen der Exzellenzstrategie der Universität Konstanz" gefördert.

Funding Open Access funding enabled and organized by Projekt DEAL.

Open Access Dieser Artikel wird unter der Creative Commons Namensnennung 4.0 International Lizenz veröffentlicht, welche die Nutzung, Vervielfältigung, Bearbeitung, Verbreitung und Wiedergabe in jeglichem Medium und Format erlaubt, sofern Sie den/die ursprünglichen Autor(en) und die Quelle ordnungsgemäß nennen, einen Link zur Creative Commons Lizenz beifügen und angeben, ob Änderungen vorgenommen wurden.

Die in diesem Artikel enthaltenen Bilder und sonstiges Drittmaterial unterliegen ebenfalls der genannten Creative Commons Lizenz, sofern sich aus der Abbildungslegende nichts anderes ergibt. Sofern das betreffende Material nicht unter der genannten Creative Commons Lizenz steht und die betreffende Handlung nicht nach gesetzlichen Vorschriften erlaubt ist, ist für die oben aufgeführten Weiterverwendungen des Materials die Einwilligung des jeweiligen Rechteinhabers einzuholen.

Weitere Details zur Lizenz entnehmen Sie bitte der Lizenzinformation auf http://creativecommons.org/ licenses/by/4.0/deed.de.

Interessenkonflikt T. Kärner, M. Goller, C. Bonnes und E. Maué geben an, dass kein Interessenkonflikt besteht.

\section{Literatur}

Abs, H.J., Döbrich, P., Gerlach-Jahn, A., \& Klieme, E. (2009). Pädagogische Entwicklungsbilanzen an Studienseminaren (PEB-Sem). Auswahl und statistische Analyse der Erhebungsinstrumente. Frankfurt a. M.: GFPF, DIPF.

Affolter, B. (2019). Engagement und Beanspruchung von Lehrpersonen in der Phase des Berufseintritts: Die Bedeutung von Zielorientierungen, Selbstwirksamkeitserwartungen und Persönlichkeitsmerkmalen im JD-R Modell. Bad Heilbrunn: Klinkhardt.

Anspal, T., Leijen, Ä., \& Löfström, E. (2019). Tensions and the teacher's role in student teacher identity development in primary and subject teacher curricula. Scandinavian Journal of Educational Research, 63(5), 679-695.

Asparouhov, T., \& Muthén, B. (2019). Latent variable interactions using maximum-likelihood and Bayesian estimation for single- and two-level models. Mplus Web Notes: No. 23. Los Angeles: Muthén \& Muthén.

Bauer, J. (2006). Prinzip Menschlichkeit: Warum wir von Natur aus kooperieren. Hamburg: Hoffmann und Campe.

Bellenberg, G., \& Thierack, A. (2003). Ausbildung von Lehrerinnen und Lehrern in Deutschland: Bestandsaufnahme und Reformbestrebungen. Opladen: Leske + Budrich.

Beuchel, P., \& Cramer, C. (2021). Beanspruchung und Achtsamkeit im Referendariat: Theoretische Einbettung des Konzepts Achtsamkeit in den Diskurs zur Beanspruchung in der Lehrerbildung. Pädagogische Rundschau, 75(4), 425-436.

Bölting, F.-J., \& Thomas, S. (2007). Das Referendariat als Ausbildungsphase der Berufseinführung. In A. Óhidy, E. Terhart \& J. Zsolnai (Hrsg.), Lehrerbild und Lehrerbildung: Praxis und Perspektiven der Lehrerausbildung in Deutschland und Ungarn (S. 203-217). Wiesbaden: Springer VS.

Bovet, G., \& Frommer, H. (2001). Praxis Lehrerberatung - Lehrerbeurteilung: Konzepte für Ausbildung und Schulaufsicht. Baltmannsweiler: Schneider.

Braun, A. (2017). Erleben Lehrkräfte und Referendare berufsbezogene Belastungen anders? Berufs(phasen) spezifische Präventionsansätze zur Gesundheitsförderung. Bad Heilbrunn: Klinkhardt.

Cain, T. (2009). Mentoring trainee teachers: how can mentors use research? Mentoring \& Tutoring: Partnership in Learning, 17(1), 53-66.

Chaplain, R.P. (2008). Stress and psychological distress among trainee secondary teachers in England. Educational Psychology, 28(2), 195-209. 
Christ, O., Van Dick, R., \& Wagner, U. (2004). Belastung und Beanspruchung bei Lehrern in der Ausbildung. In A. Hillert \& E. Schmitz (Hrsg.), Psychosomatische Erkrankungen bei Lehrerinnen und Lehrern (S. 113-119). Stuttgart: Schattauer.

Christ, O. (2004). Die Überprüfung der transaktionalen Stresstheorie im Lehramtsreferendariat. Dissertation, Philipps-Universität Marburg. https:/www.researchgate.net/profile/Oliver_Christ/publication/ 36723280_Die_Uberprufung_der_transaktionalen_Stresstheorie_im_Lehramtsreferendariat/ links/0f317531987528d2d2000000/Die-Ueberpruefung-der-transaktionalen-Stresstheorie-im-Lehr amtsreferendariat.pdf. Zugegriffen: 15. Sept. 2019.

Colquitt, J. A. (2001). On the dimensionality of organizational justice: a construct validation of a measure. Journal of Applied Psychology, 86, 386-400.

Darius, S., Bunzel, K., Ehms-Ciechanowicz, E., \& Böckelmann, I. (2020). Psychische Gesundheit bei Referendaren. Prävention und Gesundheitsförderung. https://doi.org/10.1007/s11553-020-00809-6.

Demerouti, E., Bakker, A. B., Nachreiner, F., \& Schaufeli, W. B. (2001). The job demands-resources model of burnout. Journal of Applied Psychology, 86(3), 499-512.

Dicke, T., Holzberger, D., Kunina-Habenicht, O., Linninger, C., Schulze-Stocker, F., Seidel, T., Terhart, E., Leutner, D., \& Kunter, M. (2016). „Doppelter Praxisschock“ auf dem Weg ins Lehramt? Verlauf und potenzielle Einflussfaktoren emotionaler Erschöpfung während des Vorbereitungsdienstes und nach dem Berufseintritt. Psychologie in Erziehung und Unterricht, 63(4), 244-257.

Donat, M., Radant, M., \& Dalbert, C. (2017). Psychologie der Schülerpersönlichkeit. In M. K. W. Schweer (Hrsg.), Lehrer-Schüler-Interaktion. Inhaltsfelder, Forschungsperspektiven und methodische Zugänge (S. 167-189). Wiesbaden: Springer.

Doyle, W. (2006). Ecological approaches to classroom management. In C. M. Evertson \& C.S. Weinstein (Hrsg.), Handbook of classroom management: Research, practice, and contemporary issues (S. 97-125). Mahwah: Lawrence Erlbaum.

Drüge, M., Schleider, K., \& Rosati, A.-S. (2014). Psychosoziale Belastungen im Referendariat - Merkmale, Ausprägungen, Folgen. Die Deutsche Schule, 106(4), 358-372.

Ferguson, S. (2017). Ask not what your mentor can do for you...: the role of reciprocal exchange in maintaining student-teacher mentorships. Sociological Forum, 33(1), 211-233.

FIBS (Fortbildung in bayerischen Schulen) (2020). Lehrgang A462-0/20/259223-1: Ein guter Seminarlehrer, Betreuungslehrer sein - ganz einfach? Gruppen-Supervision für Seminarlehrer, Betreuungslehrer. https://fibs.alp.dillingen.de/suche/details.php?v_id=205975. Zugegriffen: 8. Dez. 2020.

Gardner, S. (2010). Stress among prospective teachers: a review of the literature. Australian Journal of Teacher Education, 35(8), 18-28.

Hair, J.F., Black, W.C., Babin, B.J., \& Anderson, R.E. (2018). Multivariate data analysis (8. Aufl.). Harlow: Pearson.

Harder, P. (2014). Werthaltungen und Ethos von Lehrern. Empirische Studie zu Annahmen über den „,guten“ Lehrer. Bamberg: University of Bamberg Press. https://fis.uni-bamberg.de/handle/uniba/2913. Zugegriffen: 14. Febr. 2021.

Harmsen, R., Helms-Lorenz, M., Maulana, R., \& Van Veen, K. (2018). The relationship between beginning teachers' stress causes, stress responses, teaching behaviour and attrition. Teachers and Teaching, 24(6), 626-643.

Heid, H. (1992). Was „leistet“ das Leistungsprinzip? Zeitschrift für Berufs- und Wirtschaftspädagogik, $88(2), 91-108$.

Heinrich, C. (2011). „,Was denkt ein New Yorker, wenn er in einen Hamburger beißt? “ Mikrophänomenologie der Macht am Beispiel des Referendariats. Wien: Passagen Verlag.

Helsper, W. (2004). Antinomien, Widersprüche, Paradoxien: Lehrerarbeit - ein unmögliches Geschäft? Eine strukturtheoretisch-rekonstruktive Perspektive auf das Lehrerhandeln. In B. Koch-Priewe, F.U. Kolbe \& J. Wildt (Hrsg.), Grundlagenforschung und mikrodidaktische Reformansätze zur Lehrerbildung (S. 49-99). Bad Heilbrunn: Klinkhardt.

Helsper, W., \& Hummerich, M. (2014). Die Lehrer-Schüler-Beziehung. In C. Tillack, N. Fischer, D. Raufelder \& J. Fetzer (Hrsg.), Beziehungen in Schule und Unterricht. Teil 1. Theoretische Grundlagen und praktische Gestaltungen pädagogischer Beziehungen (S. 32-59). Immenhaus: Prolog.

Helsper, W., \& Wiezorek, C. (2006). Zwischen Leistungsforderung und Fürsorge. Perspektiven der Hauptschule im Dilemma von Fachunterricht und Unterstützung. Die Deutsche Schule, 98(4), 436-455.

Hennissen, P., Crasborn, F., Brouwer, N., Korthagen, F., \& Bergen, T. (2008). Mapping mentor teachers' roles in mentoring dialogues. Educational Research Review, 3(2), 168-186.

Heymann, H. W. (2009). Praxis bildet - aber wie? Gelingensbedingungen für Praxisphasen und Vorbereitungsdienst. Pädagogik, 61(9), 6-10. 
Hintz, D. (2000). Leistungsbeurteilung als pädagogisches Handeln - in der 2. Phase der Lehrerausbildung. In I. E. Cloer, D. Klika \& H. Kunert (Hrsg.), Welche Lehrer braucht das Land? Notwendige und mögliche Reformen der Lehrerbildung (S. 282-295). Weinheim: Juventa.

Hofer, M., Pekrun, R., \& Zielinski, W. (1986). Die Psychologie des Lerners. In B. Weidenmann, A. Krapp, M. Hofer, G. L. Huber \& H. Mandl (Hrsg.), Pädagogische Psychologie (S. 219-276). Weinheim: Psychologie Verlags Union.

Horstmeyer, J. (2018). Personen- und Organisationsmerkmale als Gelingensbedingungen im pädagogischen Vorbereitungsdienst. Eine Sekundäranalyse auf der Grundlage der Pädagogischen Entwicklungsbilanzen an Studienseminaren (PEB-Sem) in Hessen. Dissertation, Johann Wolfgang GoetheUniversität zu Frankfurt am Main. http://publikationen.ub.uni-frankfurt.de/frontdoor/index/index/ docId/47022. Zugegriffen: 11. Nov. 2020.

Jones, E.E., \& Pittman, T.S. (1982). Toward a general theory of strategic self-presentation. In J. Suls (Hrsg.), Psychological perspectives on the self (S. 231-262). Hillsdale: Erlbaum.

Kanders, M. (2000). Das Bild der Schule aus der Sicht der Schüler und Lehrer II. Dortmund: IFS.

Kärner, T., Bonnes, C., \& Schölzel, C. (2018). Konstruktives Feedback und nachvollziehbare Leistungserwartungen? Analysen zur wahrgenommenen Bewertungstransparenz im Referendariat. Bildung und Beruf, 1(8), 108-114.

Kärner, T., Bonnes, C., \& Schölzel, C. (2019). Bewertungstransparenz im Referendariat. Zeitschrift für Pädagogik, 65(3), 378-400.

Kärner, T., Bottling, M., Friederichs, E., \& Sembill, D. (2021a). Between adaptation and resistance: a study on resilience competencies, stress, and well-being in German VET teachers. Frontiers in Psychology, 12(619912), 1-16.

Kärner, T., Bonnes, C., Maué, E., Goller, M., \& Schmidt, V. (2021b). Transparenz, Fairness, Vertrauen und Ambivalenz im Vorbereitungsdienst: Entwicklung eines Instruments zur Charakterisierung der professionellen pädagogischen Beziehung zwischen angehenden Lehrpersonen und deren Ausbildungslehrkräften. In E. Wittmann, D. Frommberger \& U. Weyland (Hrsg.), Jahrbuch der berufs- und wirtschaftspädagogischen Forschung 2021 (S. 85-104). Opladen: Barbara Budrich.

Kaststaller, M., Hagenauer, G., \& Hascher, T. (2014). Unterschiede im emotionalen Belastungserleben und im Unterrichtsverhalten als ein Resultat problematischer LehrerInnen-SchülerInnen-Beziehungen? Ergebnisse einer empirischen Untersuchung mit österreichischen Gymnasiallehrkräften. https://boris. unibe.ch/97374/. Zugegriffen: 18. Jan. 2020.

Kentzer, N., Bracey, P., \& Devecchi, C. (2019). Using activity theory and Q methodology to examine the experiences of in-service trainee teachers in the English further education sector. Research in PostCompulsory Education, 24(4), 439-461.

Kiper, H. (2014). Beziehungen in Schule und Unterricht. In C. Tillack, N. Fischer, D. Raufelder \& J. Fetzer (Hrsg.), Beziehungen in Schule und Unterricht. Teil 1. Theoretische Grundlagen und praktische Gestaltungen pädagogischer Beziehungen (S. 11-31). Immenhaus: Prolog.

Klein, A., \& Moosbrugger, H. (2000). Maximum likelihood estimation of latent interaction effects with the LMS method. Psychometrika, 65(4), 457-474.

Klusmann, U., Kunter, M., Voss, T., \& Baumert, J. (2012). Berufliche Beanspruchung angehender Lehrkräfte: Die Effekte von Persönlichkeit, pädagogischer Vorerfahrung und professioneller Kompetenz. Zeitschrift für Pädagogische Psychologie, 26(4), 275-290.

Košinár, J. (2013). Das Problem asymmetrischer Beziehungen für Kooperation im Referendariat. In M. Keller-Schneider, S. Albisser \& J. Wissinger (Hrsg.), Professionalität und Kooperation in Schulen. Beiträge zur Diskussion über Schulqualität (S. 227-244). Bad Heilbrunn: Klinkhardt.

Košinár, J. (2014). Typenspezifischer Umgang mit den Anforderungen des Referendariats. Lehrerbildung auf dem Prüfstand, 7(2), 120-137.

Krautz, J., \& Schieren, J. (2013). Persönlichkeit und Beziehung als Grundlage der Pädagogik. Zur Einführung. In J. Krautz \& J. Schieren (Hrsg.), Persönlichkeit und Beziehung als Grundlage der Pädagogik (S. 7-27). Weinheim: Beltz.

Krüger, J. (2014). Perspektiven Pädagogischer Professionalisierung: Lehrerbildner/-innen im Vorbereitungsdienst für das Lehramt an beruflichen Schulen. Wiesbaden: Springer VS.

Kruse-Moosmayer, H., \& Zoller, U. (2007). Wege zur Gestaltung der Seminararbeit - Kooperation als Grundlage für Lernprozesse. In P. Daschner \& U. Drews (Hrsg.), Kursbuch Referendariat (S. 88-101). Weinheim: Beltz.

Kultusministerkonferenz (2018). Standards für die Lehrerbildung: Bildungswissenschaften. https://www. kmk.org/fileadmin/veroeffentlichungen_beschluesse/2004/2004_12_16-Standards-LehrerbildungBildungswissenschaften.pdf. Zugegriffen: 13. Apr. 2020 
Kupila, P., Ukkonen-Mikkola, T., \& Rantala, K. (2017). Interpretations of mentoring during early childhood education mentor training. Australian Journal of Teacher Education, 42(10), 36-49.

Kyriacou, C. (2001). Teacher stress: directions for future research. Educational Review, 53(1), $27-35$.

Kyriacou, C., \& Sutcliffe, J. (1978). Teacher stress: prevalence, sources, and symptoms. British Journal of Educational Psychology, 48(2), 159-167.

Laireiter, A., \& Lettner, K. (1993). Belastende Aspekte Sozialer Netzwerke und Sozialer Unterstützung: Ein Überblick über den Phänomenbereich und die Methodik. In A. Laireiter (Hrsg.), Soziales Netzwerk und soziale Unterstützung: Konzepte, Methoden und Befunde (S. 101-111). Bern: Huber.

Lazarus, R. S. (1966). Psychological stress and coping process. New York: McGraw-Hill.

Lazarus, R. S., \& Folkman, S. (1984). Stress, appraisal, and coping. New York: Springer.

Lenhard, H. (2004). Zweite Phase an Studienseminaren und Schulen. In S. Blömke, P. Reinholf, G. Tulodziecki \& J. Wildt (Hrsg.), Handbuch Lehrerbildung (S. 275-290). Bad Heilbrunn: Klinkhardt.

Leshem, S. (2012). The many faces of mentor-mentee relationships in a pre-service teacher education programme. Creative Education, 3(4), 413-421.

Little, T.D., Bovaird, J.A., \& Widaman, K.F. (2006). On the merits of orthogonalizing powered and product terms: implications for modeling interactions among latent variables. Structural Equation Modeling, 13(4), 497-519.

Lohaus, A., Beyer, A., \& Klein-Heßling, J. (2004). Stresserleben und Stresssymptomatik bei Kindern und Jugendlichen. Zeitschrift für Entwicklungspsychologie und Pädagogische Psychologie, 36(1), 38-46.

Lohaus, A., Eschenbeck, H., Kohlmann, C. W., \& Klein-Heßling, J. (2018). Fragebogen zur Erhebung von Stress und Stressbewältigung im Kindes- und Jugendalter - Revision (SSKJ 3-8). Göttingen: Hogrefe.

Looser, D. (2019). Die Bedeutung der Lehrer-Schüler-Beziehung für die Lern- und Leistungsmotivation von Schülern. Erziehungskompetente Lehrer aus der Perspektive der Selbstbestimmungs- und Erziehungsstiltheorie. In U. Herrmann (Hrsg.), Pädagogische Beziehungen: Grundlagen - PraxisformenWirkungen (S. 100-112). Weinheim: Beltz.

Maier, G. W., Streicher, B., Jonas, E., \& Woschée, R. (2007). Gerechtigkeitseinschätzungen in Organisationen. Die Validität einer deutschsprachigen Fassung des Fragebogens von Colquitt (2001). Diagnostica, 53(2), 97-108.

Manning, C., \& Hobson, A.J. (2017). Judgemental and developmental mentoring in further education initial teacher education in England: mentor and mentee perspectives. Research in Post-Compulsory Education, 22(4), 574-595.

Marsh, H. W., Wen, Z., Hau, K.-T., Little, T. D., Bovaird, J. A., \& Widaman, K. F. (2007). Unconstrained structural equation models of latent interactions: Contrasting residual- and mean-centered approaches. Structural Equation Modeling, 14(4), 570-580.

Maslach, C., \& Jackson, S.E. (1981). Maslach burnout inventory, manual. Palo Alto: Consulting Psychologists Press.

Maslowsky, J., Jager, J., \& Hemken, D. (2015). Estimating and interpreting latent variable interactions: a tutorial for applying the latent moderated structural equations method. International Journal of Behavioral Development, 39(1), 87-96.

Menge, C., \& Schaeper, H. (2019). Berufliche Selbstregulation von Lehrkräften: Überprüfung eines Kurzinstruments. Zeitschrift für Erziehungswissenschaft, 22(6), 1489-1513.

Munderloh, O. (2018). Das Referendariat aus der Sicht der Referendar/innen. Eine ländervergleichende Studie der zweiten Phase der Lehrerausbildung an berufsbildenden Schulen. Weinheim: Beltz.

Oser, F. (1996). Wann lernen Lehrer ihr Berufsethos? In A. Leschinsky (Hrsg.), Die Institutionalisierung von Lehren und Lernen. Beiträge zu einer Theorie der Schule (Zeitschrift für Pädagogik, 34. Beiheft: S. 235-243). Weinheim: Beltz.

Pinnick, S. (2020). Mentoring secondary English trainee teachers: a case study. English in Education, 54(3), 251-264.

Prengel, A. (2019). Pädagogische Beziehungen zwischen Anerkennung, Verletzung und Ambivalenz. Opladen: Barbara Budrich.

R Core Team (2021). R: A language and environment for statistical computing. R Foundation for Statistical Computing, Vienna, Austria. https://www.R-project.org/. Zugegriffen: 12. Mai 2021.

Richter, D., Kunter, M., Lüdtke, O., Klusmann, U., \& Baumert, J. (2011). Soziale Unterstützung beim Berufseinstieg ins Lehramt. Eine empirische Untersuchung zur Bedeutung von Mentoren und Mitreferendaren. Zeitschrift für Erziehungswissenschaft, 14(1), 35-59.

Rosseel, Y. (2012). lavaan: an R package for structural equation modeling. Journal of Statistical Software, 48(2), 1-36.

Rudow, B. (1994). Die Arbeit des Lehrers. Bern: Huber. 
Rudow, B. (2000). Der Arbeits-und Gesundheitsschutz im Lehrerberuf: Gefährdungsbeurteilung der Arbeit von Lehrerinnen und Lehrern. Ludwigsburg: Süddeutscher Pädagogischer Verlag.

Sauro, M.D., Jorgensen, R.S., \& Pedlow, C.T. (2003). Stress, glucocorticoids, and memory: a meta-analytic review. Stress, 6(4), 235-245.

Schubarth, W., Speck, K., \& Seidel, A. (2007). Endlich Praxis! Die zweite Phase der Lehrerbildung. Potsdamer Studien zum Referendariat. Frankfurt a. M.: Peter Lang.

Schulte, M. (2008). Das Studienseminar. Die Ausbildung im Studienseminar (Gymnasium \& Gesamtschule) aus der Perspektive der Referendare. Dissertation, Universität Siegen. https://dokumentix.ub.unisiegen.de/opus/volltexte/2008/365/pdf/DruckversionDiss- Schulte.pdf. Zugegriffen: 25. Nov. 2019.

Schumann, S. (2019). Belastungserleben von angehenden Lehrpersonen der Sekundarstufe II in der Schweiz und in Deutschland. In D. Holtsch, M. Oepke \& S. Schumann (Hrsg.), Lehren und Lernen auf der Sekundarstufe II. Gymnasial- und wirtschaftspädagogische Perspektiven (S. 430-440). Bern: hep.

Schweer, M. K. W. (1996). Vertrauen in der pädagogischen Beziehung. Bern: Huber.

Schweer, M. K. W. (2008). Vertrauen im Klassenzimmer. In M. K. W. Schweer (Hrsg.), Lehrer-Schüler-Interaktion. Inhaltsfelder, Forschungsperspektiven und methodische Zugänge (S. 546-564). Wiesbaden: Springer VS.

Schweer, M. K. W. (2012). Vertrauen als zentrale Ressource der Organisationsberatung: Ausgewählte empirische Befunde zu Vertrauenskulturen und Innovationsmanagement. In H. Möller (Hrsg.), Vertrauen in Organisationen: Riskante Vorleistung oder hoffnungsvolle Erwartung (S. 63-91). Wiesbaden: Springer VS.

Schweer, M.K.W., Thies, B., \& Lachner, R.P. (2017). Soziale Wahrnehmungsprozesse und unterrichtliches Handeln. Eine dynamisch transaktionale Perspektive. In M. K. W. Schweer (Hrsg.), LehrerSchüler-Interaktion. Inhaltsfelder, Forschungsperspektiven und methodische Zugänge (S. 121-145). Wiesbaden: Springer VS.

Sembill, D. (1992). Problemlösefähigkeit, Handlungskompetenz und Emotionale Befindlichkeit. Zielgrößen Forschenden Lernens. Göttingen: Hogrefe.

Sembill, D. (1995). Der Wille zum Nicht-Müssen - Gestaltungskraft im Spannungsverhältnis von Innovation und Organisation. In G.P. Bunk \& R. Lassahn (Hrsg.), Festschrift für Artur Fischer zum 75. Geburtstag (S. 125-146). Steinbach bei Gießen: Ehgart \& Albohn.

Sembill, D. (2012). Was bedeutet die Hirnforschung für die Schul- und Lernkultur? In J. Warwas, P. Harder \& D. Sembill (Hrsg.), Kultur der Schule - Schule der Kultur(en)? (S. 85-112). Baltmannsweiler: Schneider Hohengehren.

Sembill, D., \& Kärner, T. (2018). Bewertung und Ausbalancierung - Heuristiken für onto- und soziogenetische Schichtungsmodellierungen in der Bildungsforschung. In M. Huber \& S. Krause (Hrsg.), Bildung und Emotion (S. 169-194). Wiesbaden: Springer VS.

Sembill, D., \& Kärner, T. (2020). Emotionen sind Macht. Analoge und digitale Rhythmen in der Fortschrittsdebatte der beruflichen Lehrpersonenbildung. In K. Heinrichs, K. Kögler \& C. Siegfried (Hrsg.), Berufliches Lehren und Lernen: Grundlagen, Schwerpunkte und Impulse wirtschaftspädagogischer Forschung. Digitale Festschrift für Eveline Wuttke zum 60. Geburtstag. bwp@ Berufsund Wirtschaftspädagogik - online (S. 1-49). https://www.bwpat.de/profil6_wuttke/sembill_kaerner_ profil6.pdf. Zugegriffen: 12.05.2021.

Semmer, N. K., Grebner, S., \& Jacobshagen, N. (2006). Diagnostik von Stress und Belastung in der Arbeit. In F. Petermann \& M. Eid (Hrsg.), Handbuch der Psychologischen Diagnostik (S. 747-753). Göttingen: Hogrefe.

Speck, K., Schubarth, W., \& Seidel, A. (2007). Theorie-Praxis-Verhältnis in der zweiten Phase der Lehrerbildung. Empirische Befunde und theoretische Implikationen. Lehrerbildung, LLF-Berichte, 22, $5-26$.

Stefaniak, A.R., Blaxton, J.M., \& Bergeman, C.S. (2021). Age differences in types and perceptions of daily stress. The International Journal of Aging and Human Development, 19, 914150211001588.

Strietholt, R., \& Terhart, E. (2009). Referendare beurteilen. Eine explorative Analyse von Beurteilungsinstrumenten in der Zweiten Phase der Lehrerbildung. Zeitschrift für Pädagogik, 55(4), 622-645.

Terhart, E. (2000). Perspektiven der Lehrerbildung in Deutschland: Abschlussbericht der von der Kultusministerkonferenz eingesetzten Kommission. Weinheim: Beltz.

Thiel, F., Richter, S. G., \& Ophardt, D. (2012). Steuerung von Übergängen im Unterricht. Eine ExpertenNovizen-Studie zum Klassenmanagement. Zeitschrift für Erziehungswissenschaft, 15(4), 727-752.

Thompson, S., Clarke, E., Quickfall, A., \& Glazzard, J. (2020). Averting the crisis in trainee teacher wellbeing - learning lessons across European contexts: a comparative study. Journal of Comparative and International Higher Education, 12(2), 38-56. 
Van Dick, R. (1999). Stress und Arbeitszufriedenheit im Lehrerberuf: Eine Analyse von Belastung und Beanspruchung im Kontext sozialpsychologischer, klinisch-psychologischer und organisationspsychologischer Konzepte. Marburg: Tectum.

Van Dick, R., \& Stegmann, S. (2013). Belastung, Beanspruchung und Stress im Lehrerberuf - Theorien und Modelle. In M. Rothland (Hrsg.), Belastung und Beanspruchung im Lehrerberuf. Modelle, Befunde, Interventionen (S. 43-59). Wiesbaden: Springer VS.

Warwas, J., Neubauer, J., \& Panzer, E. (2016). Unterstützung und Beanspruchung im Referendariat aus der Perspektive angehender Berufsschullehrkräfte. Zeitschrift für Berufs- und Wirtschaftspädagogik, 112(2), 294-313.

Weiß, S., Schlotter, P., \& Kiel, E. (2014). Das Referendariat - Eine Zeit „schwieriger Beziehungen“? In C. Tillack, N. Fischer, D. Raufelder \& J. Fetzer (Hrsg.), Beziehungen in Schule und Unterricht. Teil 1. Theoretische Grundlagen und praktische Gestaltungen pädagogischer Beziehungen (S. 148-167). Immenhaus: Prolog.

Wernet, A. (2009). Konformismus statt kollegiale Anerkennung: Fallstudien zur Ausbildungskultur im Referendariat am Beispiel von Beurteilungen. Pädagogische Korrespondenz, 39(9), 46-63.

Wirtz, M. A., \& Strohmer, J. (2017). Dorsch Lexikon der Psychologie. Göttingen: Hogrefe.

Wolf, I. A. (1998). Effekte von Stress, sozialer Unterstützung und Persönlichkeitsvariablen auf psychisches Befinden. Dissertation, Philipps-Universität Marburg. http://archiv.ub.uni-marburg.de/diss/z1998/ 0120/pdf/diw.pdf. Zugegriffen: 20. Jan. 2020.

Wolff, C.E., Jarodzka, H., Van den Bogert, N., \& Boshuizen, H.P. A. (2016). Teacher vision: expert and novice teachers' perception of problematic classroom management scenes. Instructional Science, 44(3), 243-265.

Xanthopoulou, D., Bakker, A. B., Demerouti, E., \& Schaufeli, W. B. (2007). The role of personal resources in the job demands-resources model. International Journal of Stress Management, 14(2), 121-141.

Yuan, K.-H., \& Bentler, P. M. (2000). Three Likelihood-based methods for mean and covariance structure analysis with nonnormal missing data. Sociological Methodology, 30(1), 165-200.

Ziegler, R. (2010). Ambiguität und Ambivalenz in der Psychologie. Begriffsverständnis und Begriffsverwendung. Zeitschrift für Literaturwissenschaft und Linguistik, 40(2), 125-171.

Zsargo, E., \& Palmer, J.F. (2019). Common understanding or 'hodgepodge'? The consistency and accuracy of school-based mentors' assessment of trainee primary teachers in England. Teacher Education Advancement Network Journal, 11(2), 72-81. 